ULB-TH-01/21

Bicocca FT-01-14

DCPT- $01 / 55$

hep-th/0106235

\title{
Brane fusion in the bosonic string and the emergence of fermionic strings
}

\author{
François Englert, ${ }^{a \text {, }}$ Laurent Houart ${ }^{b, \text {,f }}$ and Anne Taormina ${ }^{c, \text { 队 }}$ \\ a Service de Physique Théorique \\ Université Libre de Bruxelles, Campus Plaine, C.P.225 \\ Boulevard du Triomphe, B-1050 Bruxelles, Belgium \\ ${ }^{b}$ Dipartimento di Fisica \\ Università Milano Bicocca \\ Piazza della Scienza 3, Milano, Italy. \\ ${ }^{c}$ Department of Mathematical Sciences \\ University of Durham \\ South Road, DH1 3LE Durham, England
}

\begin{abstract}
We review the emergence of the ten-dimensional fermionic closed string theories from subspaces of the Hilbert space of the 26-dimensional bosonic closed string theory compactified on an $E_{8} \times S O(16)$ lattice. They arise from a consistent truncation procedure which generates space-time fermions out of bosons. This procedure is extended to open string sectors. We prove that truncation of the unique tadpolefree $S O\left(2^{13}\right)$ bosonic string theory compactified on the above lattice determines the anomaly free Chan-Paton group of the Type I theory and the consistent Chan-Paton groups of Type $\mathrm{O}$ theories. It also predicts the tension of space-filling D-branes in these fermionic theories. The derivation of these fermionic string properties from bosonic considerations alone points towards a dynamical origin of the truncation process. Space-time fermions and supersymmetries would then arise from bosonic degrees of freedom and no fermionic degrees of freedom would be needed in a fundamental theory of quantum gravity.
\end{abstract}

\footnotetext{
${ }^{1}$ E-mail : fenglert@ulb.ac.be

${ }^{2}$ E-mail : Laurent.Houart@mib.infn.it

${ }^{3}$ E-mail : anne.taormina@durham.ac.uk
} 


\section{Introduction}

It has been demonstrated previously that all the ten-dimensional closed superstring theories (Type IIA, Type IIB and the two distinct heterotic superstrings) are hidden in the Hilbert space of the 26-dimensional closed bosonic string theory [1]. More precisely, the states and interaction vertices defining perturbatively these superstrings in the light-cone gauge form a subset of states and interaction vertices of the bosonic string. The emergence of space-time fermions and of supersymmetry, anticipated by Freund [2], is an impressive property of the bosonic string. The generation of space-time fermions out of bosons appears in reference [1] as a stringy generalisation of the field theoretical mechanism by which non abelian monopoles become fermions in an appropriate environment [3].

The superstrings arise from a toroidal compactification of the bosonic strings on an $E_{8} \times E_{8}$ lattice where only the second $E_{8}$ plays an active rôle. The superstring content of the bosonic string appears when all states pertaining to the first $E_{8}$ are removed from the spectrum. We call this a "truncation". The truncation required to reveal superstrings has in fact to be extended to all oscillator states in four of the eight compact dimensions spanned by the second $E_{8}$ lattice of zero modes. However, some particular zero modes have to be retained in these dimensions. They play an essential rôle in the construction and have an interesting interpretation in terms of superghost zero modes.

The $E_{8}$ lattice is a sublattice of the $S O(16)$ lattice and the theory is more elegantly formulated in terms of $E_{8} \times S O(16)$ [4]. This formulation was in fact a crucial step, because it led to uncover not only the superstrings, but also the non-supersymmetric fermionic stringst. Type OA and Type OB [5] and all the consistent non-supersymmetric ten-dimensional heterotic strings discovered in reference [6] emerged indeed from the 26-dimensional bosonic string compactified on an $E_{8} \times S O(16)$ lattice, using the same truncation that generated the superstrings out of its $E_{8} \times E_{8}$ sublattice [7, 8, 9].

The universality of the truncation procedure, leading from the bosonic string

\footnotetext{
${ }^{1}$ We shall call fermionic strings all strings containing space-time fermions in separate left or right sectors whether or not they are projected out in the closed string spectrum.
} 
to all these fermionic strings, suggests that this process is a dynamical one. It was in fact conjectured in reference [4] that string field expectation values in string field theory may provide the mechanism needed to move out of the tachyonic vacuum of the bosonic string to reach the vacuum of a truncated theory. This remained a pure speculation devoid of any predictive content and there was in fact no clear indication that truncation had any dynamical significance.

In this paper we extend the truncation procedure to open strings. Our basic assumption is that the bosonic string compactified on an $E_{8} \times S O(16)$ lattice (or on its $E_{8} \times E_{8}$ sublattice) must be free of massless tadpole divergences in leading order in the coupling constant. This tadpole condition is in line with the philosophy of building consistent "open-descendants" from the closed string sector [10]. There are two types of massless tadpoles in the bosonic theory: the dilaton tadpole and tadpoles associated with massless scalars originating from the group symmetry of the compactified theory (hereafter referred to as $\mathcal{G}$-scalars). Upon truncation all massless modes created by operators in non-compact dimensions become massive, and a subset of $\mathcal{G}$-scalars are transmuted to new massless modes, including a new dilaton and a new graviton. The tadpole condition survives the truncation for the transmuted modes and we obtain, from purely bosonic considerations, the correct Chan-Paton groups of the anomaly free Type I theory and of the tadpole-free type $\mathrm{O}$ theories.

These results are related to the existence of a unique open descendant of the uncompactified bosonic string satisfying the tadpole condition, namely the unoriented string with Chan-Paton group $S O\left(2^{13}\right)$. It turns out indeed that, upon compactification, the rank of this Chan-Paton group is reduced exactly to the value required for the fermionic string generated by truncation. This effect will be shown to arise from a fusion mechanism whereby many space-filling D-branes coalesce into one .

Interpretation of Chan-Paton multiplicities in terms of D-branes and orientifold leads to yet another prediction. We prove that the tensions of fermionic D9-branes arising from truncation equal the ten-dimensional tensions of the bosonic wrapped D25-brane and we check that the correct values for the

\footnotetext{
${ }^{2}$ Reduction of the rank of Chan-Paton groups in compactification has originally been discussed in reference [11].
} 
tensions of fermionic D9-branes are indeed recovered. This result may be interpreted as energy conservation in the truncation process.

The paper is organised as follows. In Section 2, we review in simple terms all the essential steps needed to understand how supersymmetric and nonsupersymmetric ten-dimensional fermionic closed string theories stem from the compactified closed bosonic string theory. In Section 3, we consider open string theories. We impose the tadpole condition on the compactified bosonic string and analyse the fusion mechanism on Lie algebra lattices. We specialise to $E_{8} \times S O(16)$ lattices and extend the truncation to open string sectors to get Type I and Type $\mathrm{O}$ theories and their D-brane tensions. In the final section 4 , we discuss the significance of our results and stress their possible relevance for a fundamental theory of quantum gravity.

\section{Truncation of the closed bosonic string}

\subsection{Fermions and world-sheet supersymmetry}

To accommodate space-time fermions in the 26-dimensional bosonic string one must meet three requirements:

a) A continuum of bosonic zero modes must be removed. This can be achieved by compactifying $d=24-s$ transverse dimensions on a $d$ dimensional torus. This leaves $s+2$ non-compact dimensions with transverse group $S O_{\text {trans }}(s)$.

b) Compactification must generate an internal group $S O_{\text {int }}(s)$ admitting spinor representations?. This can be achieved by toroidal compactification on the Lie lattice of a simply laced Lie group $\mathcal{G}$ of rank $d$ containing a subgroup $S O_{\text {int }}(s)$. The latter is then mapped onto $S O_{\text {trans }}(s)$ in such a way that the diagonal algebra $s_{\text {diag }}(s)=\operatorname{diag}\left[\operatorname{so}_{\text {trans }}(s) \times s o_{\text {int }}(s)\right]$ becomes identified with a new transverse algebra. In this way, the spinor representations of $S O_{\text {int }}(s)$ describe fermionic states because a rotation in space induces a half-angle rotation on these states. This mechanism is distinct from

\footnotetext{
${ }^{3}$ We shall designate all locally isomorphic groups by the same notation.
} 
the two-dimensional world-sheet equivalence of bosons and fermions. It is reminiscent of a similar mechanism at work in monopole theory: there, the diagonal subgroup of space-time rotations and isospin rotations can generate space-time fermions from a bosonic field condensate in spinor representations of the isospin group [3].

c) The consistency of the above procedure relies on the possibility of extending the diagonal algebra $s o_{\text {diag }}(s)$ to the new full Lorentz algebra $\operatorname{so}_{\text {diag }}(s+1,1)$, a highly non trivial constraint. To break the original Lorentz group $S O(25,1)$ in favour of the new one, a truncation consistent with conformal invariance must be performed on the physical spectrum of the bosonic string. Actually, states described by 12 compactified bosonic fields must be truncated, except possibly for zero modes. This follows from the need, in string theory, of a local world-sheet supersymmetry to accommodate spacetime fermions. In units where each two-dimensional boson contributes 1 to the Virasoro central charge, the related superghost contributes 11, while the longitudinal and time-like Majorana fermions contribute $2 \times 1 / 2$. Therefore, one requires 12 compactified bosons to account for the anomaly of the superghost and unphysical Majorana fermions. Moreover, the need to generate an internal group $S O_{i n t}(s)$ via toroidal compactification requires $s / 2$ compactified bosons which can account for $s$ transverse Majorana fermions (we hereafter take $s$ to be even, in which case $s / 2$ is the rank of the internal group). Therefore, one must ensure that the total number $d=24-s$ of compactified dimensions is at least $12+s / 2$. In other words,

$$
s \leq 8
$$

and the highest available space-time dimension accommodating fermions is therefore $s+2=10$ [1, 团.

We shall verify below that the closure of the Lorentz algebra indeed requires Eq.(11). 


\subsection{Closed string compactification and zero modes}

Consider the bosonic closed string compactified on a $d$-dimensional torus. In terms of the left and right compactified momenta, the mass spectrum is

$$
\begin{array}{rlrl}
\frac{\alpha^{\prime} m_{L}^{2}}{4} & =\alpha^{\prime} \mathbf{p}_{L}^{2}+N_{L}-1, \\
\frac{\alpha^{\prime} m_{R}^{2}}{4} & =\alpha^{\prime} \mathbf{p}_{R}^{2}+N_{R}-1, \\
m^{2}=\frac{m_{L}^{2}}{2}+\frac{m_{R}^{2}}{2} \quad & \text { and } & ; & m_{L}^{2}=m_{R}^{2} .
\end{array}
$$

In Eq.(2) $N_{L}$ and $N_{R}$ are the oscillator numbers in 26-dimensions and the zero modes $\sqrt{2 \alpha^{\prime}} \mathbf{p}_{L}, \sqrt{2 \alpha^{\prime}} \mathbf{p}_{R}$ span a $2 d$-dimensional even self-dual Lorentzian lattice with negative (resp. positive) signature for left (resp. right) momenta. This ensures modular invariance of the closed string spectrum [12]. For generic toroidal compactifications, the massless vectors $\alpha_{-1, R}^{\mu} \alpha_{-1, L}^{i}\left|0_{L}, 0_{R}\right\rangle$ and $\alpha_{-1, L}^{\mu} \alpha_{-1, R}^{i}\left|0_{L}, 0_{R}\right\rangle$, where the indices $\mu$ and $i$ respectively refer to noncompact and compact dimensions, generate a local symmetry $\left[U_{L}(1)\right]^{d} \times$ $\left[U_{R}(1)\right]^{d}$. But more massless vectors arise when $\sqrt{2 \alpha^{\prime}} \mathbf{p}_{L}$ and $\sqrt{2 \alpha^{\prime}} \mathbf{p}_{R}$ are roots of simply laced groups $\mathcal{G}_{L}$ and $\mathcal{G}_{R}$ (with roots length $\sqrt{2}$ ). The gauge symmetry is enlarged to $\mathcal{G}_{L} \times \mathcal{G}_{R}$ and the theory is modular invariant provided the lattice of zero modes is self-dual Lorentzian even. In particular, this is the case for compactification on a $\mathcal{G} \times \mathcal{G}$ lattice where $\mathcal{G}$ is any semisimple simply laced group of rank $d$, if both $\sqrt{2 \alpha^{\prime}} \mathbf{p}_{L}$ and $\sqrt{2 \alpha^{\prime}} \mathbf{p}_{R}$ span the full weight lattice $\Lambda_{\text {weight }}$ of $\mathcal{G}$, but are constrained to be in the same conjugacy class. Namely $\sqrt{2 \alpha^{\prime}}\left(\mathbf{p}_{L}-\mathbf{p}_{R}\right)$ must be on the root lattice $\Lambda_{\text {root }}$ of $\mathcal{G}$ [13]. Hereafter, such lattice will be referred to as the EN lattice of $\mathcal{G}$.

Now perform a toroidal compactification on a $\mathcal{G}_{L} \times \mathcal{G}_{R}$ lattice. Then truncate the spectrum of, say, the right sector of the theory, by tentatively removing all states (i.e. oscillators and momenta) in $24-3 s / 2$ of the total number $d=24-s$ of compact dimensions, leaving $s+2$ non-compact dimensions. Also, since the truncation removes a subset of states whose compactified momenta lie on the weight lattice of $\mathcal{G}_{R}$, one expects the embedding of the subgroup $S O_{\text {int }}(s)$ of $\mathcal{G}_{R}$ to be regular't This must in fact be the case as

\footnotetext{
${ }^{4} \mathrm{An}$ embedding is regular if the root lattice of the subgroup is contained in the root lattice of the group.
} 
discussed below.

Let us examine the closure of the Lorentz algebra $s o(s+1,1)$ 迎, (4). The generators of the diagonal group $S O_{\text {diag }}(s)$ are given by

$$
J^{i j}=L^{i j}+K_{o}^{i j}, \quad i, j=1, \ldots, s,
$$

where $L^{i j}$ are the generators of the transverse space-time group $S O_{\text {trans }}(s)$ and the operators $K_{o}^{i j}$ generate the internal $S O_{i n t}(s)$. Note that Eq.(蛋) maps the internal group onto $S O_{\text {trans }}(s)$. The $K_{o}^{i j}$ are the zero modes of the full Kac-Moody algebra generated in the compact dimensions from bosonic vertex operators and obey the commutation relations

$$
\left[K_{m}^{i j}, K_{n}^{l p}\right]=i\left(K_{m+n}^{i p} \delta^{j l}+K_{m+n}^{j l} \delta^{i p}-K_{m+n}^{i l} \delta^{j p}-K_{m+n}^{j p} \delta^{i l}\right)+2 m k \delta_{p l}^{i j} \delta_{m+n, o}
$$

where

$$
\delta_{p l}^{i j}=\frac{1}{2}\left(\delta_{p}^{i} \delta_{l}^{j}-\delta_{l}^{i} \delta_{p}^{j}\right)
$$

and $k$ is the level of the algebra. To check the closure of the Lorentz algebra, one has to supplement Eq.(四) with the generators $J^{i+}, J^{+-}$and $J^{i-}$ defined in the light-cone gauge. The first $s+1$ operators only involve position and momentum operators, but the $J^{i-}$ involve oscillators in $\nu=s / 2+s$ compact and transverse non-compact dimensions. The Kac-Moody generators Eq.(5) are needed in the $J^{i-}$ to ensure their correct commutation relations with the $J^{i j}$. It remains to show that boosts commute, i.e.

$$
\left[J^{i-}, J^{j-}\right]=0 \text {. }
$$

This is a non-trivial requirement. Imposing the mass shell condition

$$
\frac{\alpha^{\prime} m_{R}^{2}}{4}=\alpha^{\prime} \mathbf{p}_{R}^{2}[S O(s)]+N_{R}(\nu)-C,
$$

where $N_{R}(\nu)$ is the oscillator number in $\nu$ dimensions and $C$ is a parameter, one obtains Eq.(7) provided [4]

$$
\frac{\nu}{12}=2-k=2 C
$$

The solution $\nu=12, k=1, C=1 / 2$, is compatible with our truncation scheme. Recall indeed that $\nu$ counts the number of compact dimensions

\footnotetext{
${ }^{5}$ The solution $k=0, \nu=24, C=1$ has nothing to do with an internal group $S O_{\text {int }}(s)$. There is no contribution from a Kac-Moody algebra; we simply recover the original bosonic string Lorentz group for any compactification and the constant $C=1$ agrees with the mass spectrum Eq.(2). The case $k=2, \nu=C=0$ will not be discussed here.
} 
associated with $S O_{\text {int }}(s)$ as well as the $s$ transverse space-time dimensions. So, $\nu=12$ corresponds to $s=8$ and, in accordance with the central charge argument developed in section 2.1, to a truncation of twelve compactified dimensions. 5 Furthermore, compactifications on lattices of simply laced groups always generate level one Kac-Moody algebras [14], and therefore, $k=1$ is consistent with torus compactification of $S O_{i n t}(s)$. Moreover, the Dynkin index of its embedding in $\mathcal{G}_{R}$ is $k\left[S \widehat{O_{\text {int }}}(s)\right] / k\left[\widehat{\mathcal{G}_{R}}\right]$ [15]. This is equal to one, which implies that the embedding is regular.

There is however a potential problem of compatibility between the closure of the new Lorentz algebra and our truncation: setting $C=1 / 2$ in Eq.(8) is not consistent with Eq.(2) if the truncation only keeps the zero mode contribution coming from the internal symmetry group $S O_{\text {int }}(8)$. There is some subtlety with zero modes in the other compact dimensions. To clarify the issue, let us further analyse the embedding of $S O_{\text {int }}(8)$ in $\mathcal{G}_{R}$ and the twelve-dimensional truncation.

The centre of the covering group of $S O(8)$ is $Z_{2} \times Z_{2}$. Its four elements partition the weight lattice in four conjugacy classes $(o)_{8},(v)_{8},(s)_{8},(c)_{8}$ isomorphic to the root lattice. The $(o)_{8}$ lattice is the root lattice itself and contains the element $\sqrt{2 \alpha^{\prime}} \mathbf{p}_{\mathbf{o}}=(0,0,0,0)$. The $(v)_{8}$ lattice is the vector lattice whose smallest weights are eight vectors of norm one; in an orthonormal basis, these are $\sqrt{2 \alpha^{\prime}} \mathbf{p}_{\mathbf{v}}=( \pm 1,0,0,0)+$ permutations. The $(s)_{8}$ and $(c)_{8}$ lattices are spinor lattices whose smallest weights also have norm one and are the eightfold degenerate vectors $\sqrt{2 \alpha^{\prime}} \mathbf{p}_{\mathbf{s}, \mathbf{c}}=( \pm 1 / 2, \pm 1 / 2, \pm 1 / 2, \pm 1 / 2)$ with even (for class $(s)_{8}$ ) or odd (for class $\left.(c)_{8}\right)$ number of minus signs. The structure of the weight lattice of all $S O(4 \mathrm{~m})$ groups is the same: in a $2 \mathrm{~m}$-dimensional Cartesian basis, the root lattice vectors have integer components whose sum is even (and contains the element $\left.\sqrt{2 \alpha^{\prime}} \mathbf{p}_{\mathbf{o}}=\mathbf{0}\right)$. The vector $\sqrt{2 \alpha^{\prime}} \mathbf{p}_{\mathbf{v}}=( \pm 1,0 \ldots$. still has norm one but the spinors $\sqrt{2 \alpha^{\prime}} \mathbf{p}_{\mathbf{s}, \mathbf{c}}=( \pm 1 / 2, \pm 1 / 2 \ldots$.$) have norm$ increasing with $m$. The degeneracy in norm of $(v)_{8},(s)_{8}$ and $(c)_{8}$ in $S O(8)$ is rooted in the triality properties of the group, and the choice of a vector representation $\sqrt{2 \alpha^{\prime}} \mathbf{p}_{\mathbf{v}}$ is a mere convention. $\sqrt{2 \alpha^{\prime}} \mathbf{p}_{\mathbf{v}}$ is in fact defined by its mapping onto the representation of the $S O_{\text {trans }}(8)$ group as described in Eq.(1). It is this mapping which transmutes the spinors $(s)_{8}$ and $(c)_{8}$ of $S O_{\text {int }}(8)$ to space-time spinors of the Lorentz group $S O(9,1)$.

\footnotetext{
${ }^{6}$ The above derivation of the closure applies trivially when some additional dimensions of the ten-dimensional strings are compactified.
} 
In order for Eq.(2) to be compatible with Eq.(8) one must keep zero modes in the 16 compact dimensions in such a way that

$$
\alpha^{\prime} \mathbf{p}_{R}^{2}\left[\mathcal{G}_{R}\right]=\alpha^{\prime} \mathbf{p}_{R}^{2}[S O(8)]+\frac{1}{2}
$$

Note that the contribution of these zero modes to the spectrum, namely $1 / 2$, is exactly the energy required to remove the zero-point energy $(-12 / 24)$ contribution to the energy of the states taken out by the truncation.

As shown below, Eq.(10) can be satisfied by choosing

$$
\mathcal{G}_{R}=E_{8} \times S O(16),
$$

and we shall prove at the end of section 2.4 that from this choice all known ten-dimensional closed fermionic strings emerge from the same truncation scheme. We decompose $S O(16)$ in $S O^{\prime}(8) \times S O(8)$ and truncate all states created by oscillators in the 12 dimensions defined by the $E_{8} \times S O^{\prime}(8)$ root lattice. To satisfy Eq.(10), we keep only $S O(16)$ zero modes which are $S O^{\prime}(8)$ vectors of norm one. The latter are chosen as follows.

The decomposition of an $S O(16)$ lattice in terms of $S O^{\prime}(8) \times S O(8)$ lattices yields

$$
\begin{aligned}
& (o)_{16}=\left[(o)_{8^{\prime}} \oplus(o)_{8}\right]+\left[(v)_{8^{\prime}} \oplus(v)_{8}\right], \\
& (v)_{16}=\left[(v)_{8^{\prime}} \oplus(o)_{8}\right]+\left[(o)_{8^{\prime}} \oplus(v)_{8}\right], \\
& (s)_{16}=\left[(s)_{8^{\prime}} \oplus(s)_{8}\right]+\left[(c)_{8^{\prime}} \oplus(c)_{8}\right], \\
& (c)_{16}=\left[(s)_{8^{\prime}} \oplus(c)_{8}\right]+\left[(c)_{8^{\prime}} \oplus(s)_{8}\right] .
\end{aligned}
$$

The vectors of norm one in $S O^{\prime}(8)$ are the 4 -vectors $\sqrt{2 \alpha^{\prime}} \mathbf{p}_{\mathbf{v}}^{\prime}, \sqrt{2 \alpha^{\prime}} \mathbf{p}_{\mathbf{s}}^{\prime}$ and $\sqrt{2 \alpha^{\prime}} \mathbf{p}_{\mathbf{c}}^{\prime}$ described above. We choose one vector $\sqrt{2 \alpha^{\prime}} \mathbf{p}_{\mathbf{v}}^{\prime}$ and one vector $\sqrt{2 \alpha^{\prime}} \mathbf{p}_{\mathbf{s}}^{\prime}$ (or equivalently $\sqrt{2 \alpha^{\prime}} \mathbf{p}_{\mathbf{c}}^{\prime}$ ).

This gives the truncations

$$
\begin{array}{ll}
(o)_{16} \rightarrow(v)_{8} & (v)_{16} \rightarrow(o)_{8} \\
(s)_{16} \rightarrow(s)_{8} & (c)_{16} \rightarrow(c)_{8}
\end{array}
$$

or

$$
\begin{array}{ll}
(o)_{16} \rightarrow(v)_{8} & (v)_{16} \rightarrow(o)_{8}, \\
(s)_{16} \rightarrow(c)_{8} & (c)_{16} \rightarrow(s)_{8} .
\end{array}
$$


It follows from the closure of the Lorentz algebra that states belonging to the lattices $(v)_{8}$ or $(o)_{8}$ are bosons while those belonging to the spinor lattices $(s)_{8}$ and $(c)_{8}$ are space-time fermions. These zero modes ensure the truncation consistency by selecting, in the light-cone gauge, the emission vertices of the fermionic strings as subsets of the emission vertices of the bosonic string [1, 4]. They may in fact be viewed as superghosts zero modes entering emission vertices in the fermionic string]. We shall therefore refer to these zero modes as to ghost vectors. We now show that this choice of zero modes $\sqrt{2 \alpha^{\prime}} \mathbf{p}_{\mathbf{v}}^{\prime}$ and $\sqrt{2 \alpha^{\prime}} \mathbf{p}_{\mathbf{s}}^{\prime}$ (or $\sqrt{2 \alpha^{\prime}} \mathbf{p}_{\mathbf{c}}^{\prime}$ ) preserve modular invariance in the truncation.

\subsection{Modular invariance}

Consider a toroidal compactification of closed strings on a $\mathcal{G}_{L} \times \mathcal{G}_{R}$ lattice which is Lorentzian self-dual even. Instead of starting with the modular invariant partition function of this lattice, we shall analyse the modular transformation properties of the partition function of the full weight lattice $\Lambda^{*} \equiv \Lambda\left(\mathcal{G}_{L} \times \mathcal{G}_{R}\right)$. This lattice admits a coset decomposition $\Lambda^{*} / \Lambda_{11}$ with $\Lambda_{11}$ the even (but in general not self-dual) Lorentzian lattice of the vectors $\sqrt{2 \alpha^{\prime}} \mathbf{p}_{11}=\left(\sqrt{2 \alpha^{\prime}} \mathbf{p}_{1 L}, \sqrt{2 \alpha^{\prime}} \mathbf{p}_{1 R}\right)$, where $\sqrt{2 \alpha^{\prime}} \mathbf{p}_{1 L}$ and $\sqrt{2 \alpha^{\prime}} \mathbf{p}_{1 R}$ span the root lattices of $\mathcal{G}_{L}$ and $\mathcal{G}_{R}$. The coset decomposition follows from the fact that any even lattice is integral (i.e. for all pairs of lattice vectors $\mathbf{v}$ and $\mathbf{v}^{\prime}$, $\mathbf{v} . \mathbf{v}^{\prime} \in Z$ ) and that any integral lattice is a sublattice of its dual. The latter can then be decomposed into an integer number $\mathcal{N}$ of cosets. In the present case, we have

$$
\Lambda^{*}=\bigoplus_{\alpha=1}^{\mathcal{N}_{L}} \bigoplus_{\beta=1}^{\mathcal{N}_{R}} \Lambda_{\alpha \beta},
$$

where the $\mathcal{N}=\mathcal{N}_{L} \mathcal{N}_{R}$ sublattices $\Lambda_{\alpha \beta}$ are isomorphic to the lattice $\Lambda_{11} \cdot \mathcal{N}_{L}$ and $\mathcal{N}_{R}$ are equal to the order of the centres of the covering groups of $\mathcal{G}_{L}$ and $\mathcal{G}_{R}$.

The partition function of each sublattice $\Lambda_{\alpha \beta}$ factorises in left and right partition functions

$$
\gamma_{\alpha \beta}(\bar{\tau}, \tau)=\bar{\gamma}_{\alpha L}(\bar{\tau}) \gamma_{\beta R}(\tau)
$$

\footnotetext{
${ }^{7}$ For a general discussion on the mapping of the states of the truncated bosonic string to the covariant bosonic formulation of fermionic strings, see reference [16].
} 
where

$$
\gamma_{\beta R}(\tau)=\sum_{\sqrt{2 \alpha^{\prime}} \mathbf{p}_{1 R} \in \Lambda_{1 R}} \exp \left\{2 \pi i \tau\left[\alpha^{\prime}\left(\mathbf{p}_{1 R}+\mathbf{p}_{\beta R}\right)^{2}+N_{R}^{(c)}-\frac{\delta_{R}}{24}\right]\right\} .
$$

Here $\Lambda_{1 R}$ is the $\mathcal{G}_{R}$ root lattice and $\mathbf{p}_{\beta R}$ is an arbitrarily chosen vector of a sublattice $\Lambda_{\beta R}$ in the coset decomposition $\Lambda_{1 R}^{*} / \Lambda_{1 R}$, where $\Lambda_{1 R}^{*}$ is the $\mathcal{G}_{R}$ weight lattice. $N_{R}^{(c)}$ is the oscillator number in the compact dimensions and $\delta_{R}$ is the number of compact dimensions. A similar expression holds for $\bar{\gamma}_{\alpha L}(\bar{\tau})$. Clearly, if $\mathcal{G}_{R}$ can be further decomposed in a direct product of simply laced groups, $\gamma_{\beta R}(\tau)$ can be further factorised accordingly. The $S$ modular transformation $(S: \tau \rightarrow-1 / \tau)$ of each factor ${ }^{\natural}$ (e.g.)

$$
\gamma_{\beta R}(\tau)=\frac{1}{\sqrt{\mathcal{N}_{R}}} \sum_{\eta=1}^{\mathcal{N}_{R}} \exp \left(4 \pi i \alpha^{\prime} \mathbf{p}_{\beta R} \cdot \mathbf{p}_{\eta R}\right) \gamma_{\eta}\left(-\frac{1}{\tau}\right)
$$

provides a simple proof that any modular invariant closed bosonic string, compactified on a $\mathcal{G}_{L} \times \mathcal{G}_{R}$ lattice, (with $\mathcal{G}_{R}$ and/or $\mathcal{G}_{L}=E_{8} \times S O(16)$ ), yields after truncation a fermionic modular invariant ten-dimensional string.

Eq.(I8) shows that under the $S$ modular transformation the partition functions of the four sublattices $(o)_{16},(v)_{16},(s)_{16},(c)_{16}$ transform as

$$
\begin{aligned}
& \gamma_{(o)_{16}} \rightarrow \frac{1}{2}\left[\gamma_{(o)_{16}}+\gamma_{(v)_{16}}+\gamma_{(s)_{16}}+\gamma_{(c)_{16}}\right], \\
& \gamma_{(v)_{16}} \rightarrow \frac{1}{2}\left[\gamma_{(o)_{16}}+\gamma_{(v)_{16}}-\gamma_{(s)_{16}}-\gamma_{(c)_{16}}\right], \\
& \gamma_{(s)_{16}} \rightarrow \frac{1}{2}\left[\gamma_{(o)_{16}}-\gamma_{(v)_{16}}+\gamma_{(s)_{16}}-\gamma_{(c)_{16}}\right], \\
& \gamma_{(c)_{16}} \rightarrow \frac{1}{2}\left[\gamma_{(o)_{16}}-\gamma_{(v)_{16}}-\gamma_{(s)_{16}}+\gamma_{(c)_{16}}\right],
\end{aligned}
$$

as do the four $S O(8)$ partition functions $\gamma_{(o)_{8}}, \gamma_{(v)_{8}}, \gamma_{(s)_{8}}$ and $\gamma_{(c)_{8}}$. The crucial point is that our truncation Eqs.(13) or (14), together with a sign flip on the spinorial $S O(8)$ partition functions $\gamma_{(s)_{8}}$ and $\gamma_{(c)_{8}}$, commutes with $S$. We show this for the truncation with ghost vector $\sqrt{2 \alpha^{\prime}} \mathbf{p}_{\mathbf{s}}^{\prime}$ (the proof is similar for $\left.\sqrt{2 \alpha^{\prime}} \mathbf{p}_{\mathbf{c}}^{\prime}\right)$. Truncating and flipping $\gamma_{(o)_{16}}$ as well as its $S$-transform as given in Eq.(19), we get the correct $S$-transform of $\gamma_{(v)_{8}}$, as depicted in the diagram below.

\footnotetext{
${ }^{8}$ This expression is a straightforward consequence of the Poisson resummation formula and of the relations $V\left(\Lambda_{11}\right)=\sqrt{\mathcal{N}}\left(V\left(\Lambda^{*}\right)=1 / \sqrt{\mathcal{N}}\right)$. See for instance reference [16].
} 


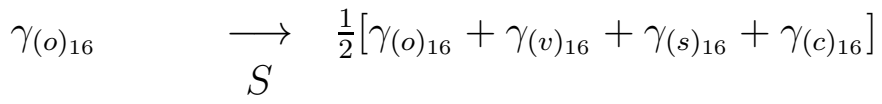

Trunc $\downarrow$ Flip $\quad$ Trunc $\downarrow$ Flip

$$
\gamma_{(v)_{8}} \quad \stackrel{S}{\longrightarrow} \frac{1}{2}\left[\gamma_{(v)_{8}}+\gamma_{(o)_{8}}-\gamma_{(s)_{8}}-\gamma_{(c)_{8}}\right]
$$

Similarly, starting with $\gamma_{(s)_{16}}$, we get the corresponding diagram.

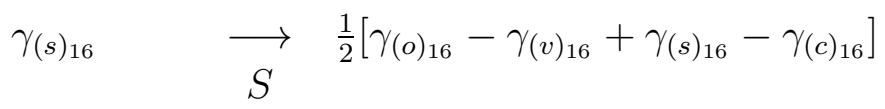

$$
\begin{array}{ccc}
\text { Trunc } \downarrow \text { Flip } & \text { Trunc } \downarrow \text { Flip } \\
& & S \\
-\gamma_{(s)_{8}} & \longrightarrow & \frac{1}{2}\left[\gamma_{(v)_{8}}-\gamma_{(o)_{8}}-\gamma_{(s)_{8}}+\gamma_{(c)_{8}}\right]
\end{array}
$$

The commutation with $S$ of the truncation-flip for the two remaining partition functions follows from the above diagrams in an obvious way. It follows from the commutation properties just described and from the factorisation of the partition function of the lattice $\Lambda^{*} \equiv \Lambda\left(\mathcal{G}_{L} \times \mathcal{G}_{R}\right)$, Eq.(16), that any $S$ invariant partition function before truncation and flip remains so afterwards.

To summarise, the truncated partition functions do have the same modular properties under inversion $S$ as the original bosonic partition functions provided, in accordance with the $S O(9,1)$ Lorentz group, internal spinors are transmuted to space-time spinors. A glance at Eq.(17) shows that the invariance under the translations $\tau \rightarrow \tau+1, \bar{\tau} \rightarrow \bar{\tau}+1$ is also preserved when both sectors are truncated. It is preserved for the heterotic strings because the half integer $\left(\delta_{R}-\delta_{L}\right) / 24=(4-16) / 24$ compensates the half integer arising from ghosts of norm one. The modular invariance of the lattice partition function ensures the modular invariance of the partition function of the full theory. This completes the proof that the truncation from $E_{8} \times S O(16)$ to $S O_{\text {int }}(8)+$ ghosts transfers modular invariance from the 26-dimensional bosonic string to ten-dimensional fermionic strings:

\footnotetext{
${ }^{9}$ For an alternate proof, see ref. [9]. Generalisation to multiloops is given in ref. [17].
} 


\subsection{Closed fermionic strings}

We now explain how the consistent fermionic ten-dimensional strings [5, 6] are obtained by truncation from the 26-dimensional bosonic string.

First we consider the fermionic theory emerging from truncation in both left and right sectors. We thus examine a compactification on both sectors with $\mathcal{G}_{L}=\mathcal{G}_{R}=E_{8} \times S O(16)$. In the truncated theory, $\left(E_{8}\right)_{L, R}$ merely disappear, and we therefore only discuss in detail the fate of the $S O(16)_{L, R}$ representations under truncation. In the full bosonic string, the representations of $S O(16)$ entering the partition function are restricted by modular invariance and right partition functions $\bar{\gamma}_{\alpha L}, \gamma_{\alpha R}$.

Using the transformation properties Eq.(19), one finds that there are two distinct modular invariant partition functions. The first one is,

$$
\begin{gathered}
\quad\left(\bar{\gamma}_{(o)_{16}, L}+\bar{\gamma}_{(s)_{16}, L}\right)\left(\gamma_{(o)_{16}, R}+\gamma_{(s)_{16}, R}\right) \\
=\bar{\gamma}_{(o)_{16}, L} \gamma_{(o)_{16}, R}+\bar{\gamma}_{(s)_{16}, L} \gamma_{(o)_{16}, R}+\bar{\gamma}_{(o)_{16}, L} \gamma_{(s)_{16}, R}+\bar{\gamma}_{(s)_{16}, L} \gamma_{(s)_{16}, R},
\end{gathered}
$$

and the second is,

$$
\bar{\gamma}_{(o)_{16}, L} \gamma_{(o)_{16}, R}+\bar{\gamma}_{(v)_{16}, L} \gamma_{(v)_{16}, R}+\bar{\gamma}_{(s)_{16}, L} \gamma_{(s)_{16}, R}+\bar{\gamma}_{(c)_{16}, L} \gamma_{(c)_{16}, R} .
$$

Eq.(20) can be rewritten as $\bar{\gamma}_{(o)_{E 8}, L} \gamma_{(o)_{E 8}, R}$ where the subscript (o) refers to the root lattice of $E_{8}$ which is a sublattice of the $S O(16)$ weight lattice. Eqs.(20) and (21) describe compactifications on the EN lattices of $E_{8} \times E_{8}$ and of $E_{8} \times S O(16)$.

To interpret the result of the truncation in conventional terms, we note that the $S O(8)$ partition functions $\gamma_{(o)_{8}}$ and $\gamma_{(v)_{8}}$, divided by the Dedekind functions arising from the bosonic states in the eight non compact transverse dimensions are the Neveu-Schwarz partition functions with the 'wrong' and 'right' GSO projection $(N S)_{-}$and $(N S)_{+}$. The partition functions $\gamma_{(s)_{8}}$ and $\gamma_{(c)_{8}}$, divided by the same Dedekind functions, form the two Ramond partition functions of opposite chirality $R_{+}$and $R_{-}$.

\footnotetext{
${ }^{10}$ Strictly speaking the modular invariance does not fully determine the representations of $S O(16)$ at this level (because $\gamma(s)=\gamma(c)$ ). However at the level of the amplitudes, this ambiguity is lifted.
} 
Choosing the ghosts $\sqrt{2 \alpha^{\prime}} \mathbf{p}_{\mathbf{v}}^{\prime}$ and $\sqrt{2 \alpha^{\prime}} \mathbf{p}_{\mathbf{s}}^{\prime}$ in both sector, and truncating in accordance with Eq.(13), the first partition function Eq.(20) yields the supersymmetric chiral closed string,

$$
\begin{gathered}
\bar{\gamma}_{(o)_{E 8}, L} \gamma_{(o)_{E 8}, R}=\bar{\gamma}_{(o)_{16}, L} \gamma_{(o)_{16}, R}+\bar{\gamma}_{(s)_{16}, L} \gamma_{(o)_{16}, R}+\bar{\gamma}_{(o)_{16}, L} \gamma_{(s)_{16}, R}+\bar{\gamma}_{(s)_{16}, L} \gamma_{(s)_{16}, R} \\
\text { Trunc } \downarrow \text { Flip } \\
\bar{\gamma}_{(v)_{8}, L} \gamma_{(v)_{8}, R}-\bar{\gamma}_{(s)_{8}, L} \gamma_{(v)_{8}, R}-\bar{\gamma}_{(v)_{8}, L} \gamma_{(s)_{8}, R}+\bar{\gamma}_{(s)_{8}, L} \gamma_{(s)_{8}, R} .
\end{gathered}
$$

Taking into account the oscillators in non compact dimensions, this is

$$
\text { IIB : } \quad(N S)_{+}(N S)_{+}+R_{+}(N S)_{+}+(N S)_{+} R_{+}+R_{+} R_{+} .
$$

Replacing $\mathbf{p}_{\mathbf{s}}^{\prime}$ by $\mathbf{p}_{\mathbf{c}}^{\prime}$ in, say, the right sector we get, using Eq.(14), the non chiral supersymmetric closed string

$$
\begin{gathered}
\bar{\gamma}_{(o)_{E 8}, L} \gamma_{(o)_{E 8}, R}=\bar{\gamma}_{(o)_{16}, L} \gamma_{(o)_{16}, R}+\bar{\gamma}_{(s)_{16}, L} \gamma_{(o)_{16}, R}+\bar{\gamma}_{(o)_{16}, L} \gamma_{(s)_{16}, R}+\bar{\gamma}_{(s)_{16}, L} \gamma_{(s)_{16}, R} \\
\text { Trunc } \downarrow \text { Flip } \\
\bar{\gamma}_{(v)_{8}, L} \gamma_{(v)_{8}, R}-\bar{\gamma}_{(s)_{8}, L} \gamma_{(v)_{8}, R}-\bar{\gamma}_{(v)_{8}, L} \gamma_{(c)_{8}, R}+\bar{\gamma}_{(s)_{8}, L} \gamma_{(c)_{8}, R} .
\end{gathered}
$$

Taking into account the oscillators in non compact dimensions, this is

$$
\text { IIA : } \quad(N S)_{+}(N S)_{+}+R_{+}(N S)_{+}+(N S)_{+} R_{-}+R_{+} R_{-} .
$$

The same choices of the ghosts in the second partition function yield the non-supersymmetric strings

$$
\begin{array}{cl}
\text { 0B }: & (N S)_{+}(N S)_{+}+(N S)_{-}(N S)_{-}+R_{+} R_{+}+R_{-} R_{-}, \\
\text {0A : } & (N S)_{+}(N S)_{+}+(N S)_{-}(N S)_{-}+R_{+} R_{-}+R_{-} R_{+} .
\end{array}
$$

Heterotic strings are generically obtained from compactification on $\mathcal{G}_{L} \times \mathcal{G}_{R}$ by only truncating in the right sector with $\mathcal{G}_{R}=E_{8} \times S O(16)$. In the general case, the partition function constructed on $\mathcal{G}_{L} \times\left[E_{8} \times S O(16)\right]_{R}$ must be modular invariant. Because of the factorization Eq.(16) we may replace the 
Lorentzian metric by a Euclidean one and drop the $E_{8}$ to preserve invariance under translation $(\tau \rightarrow \tau+1)$ in the Euclidean metric. This reduces the problem of finding all heterotic strings obtainable in this way to that of finding all 24-dimensional Euclidean even self-dual lattices containing a sublattice $\Lambda(S O(16))$. All 24-dimensional even self-dual Euclidean lattices have been classified: they are known as the Niemeier lattices. Heterotic strings are obtained from the relevant Niemeier lattices by the truncation Eq.(13) (or equivalently, by Eq.(14)) [7]. In particular, one recovers the usual supersymmetric heterotic strings when only the sublattices $(o)_{16} \oplus(s)_{16}=\Lambda\left(E_{8}\right)$ are kept in $\Lambda(S O(16))$. Indeed, this is an even self-dual Euclidean lattice, and therefore, the lattice $\Lambda\left(\mathcal{G}_{L}\right)$ must also be even self-dual Euclidean, namely $\Lambda\left(E_{8} \times E_{8}\right)$ or $\Lambda\left(\operatorname{Spin}(32) / Z_{2}\right)=(o)_{32} \oplus(s)_{32}$.

\section{$3 \quad$ Brane fusion and the open string sectors}

We now turn to the 10-dimensional open string theories: the supersymmetric Type I theory and the non-supersymmetric Type $\mathrm{O}$ theories.

We shall impose the tadpole condition on the bosonic string theory, namely we impose that massless tadpoles do not contribute to vacuum amplitudes in leading order in the coupling constant.

After reviewing the well-known result that, for the uncompactified bosonic string, this condition leads to the unique unoriented string theory with ChanPaton group $S O\left(2^{13}\right)$ [18, we study its implications for strings compactified on Lie algebra lattices. We specialise to $E_{8} \times E_{8}$ and $E_{8} \times S O(16)$ lattices, and perform the truncation to obtain the Type I theory and the Type O tadpole-free theories.

We interpret the reduction of the Chan-Paton multiplicities which led to these results in terms of orientifolds and D-branes. We make explicit the fusion mechanism whereby many space-filling D-branes coalesce into one. Fusion relates all fermionic open string theories to the ancestor bosonic $S O\left(2^{13}\right)$

\footnotetext{
${ }^{11}$ All these heterotic strings have rank 16 groups $\mathcal{G}_{L}$. There is one heterotic string with left symmetry $E_{8}$. Although it can still be derived by the general truncation procedure from the bosonic string, it involves an additional twist [8].
} 
theory.

Finally we prove that energy is conserved in the truncation process.

\subsection{The bosonic open string ancestor}

We review the derivation of the existence of a 26-dimensional open bosonic string free of massless tadpole divergences $[2$.

The Chan-Paton group of the 26-dimensional unoriented, uncompactified, open string theory may be fully determined by the tadpole condition [10, 19, 201. The full one-loop vacuum amplitude of a theory with open and closed unoriented strings comprises the four loop amplitudes with vanishing Euler characteristic: the torus $\mathcal{T}$, the Klein bottle $\mathcal{K}$, the annulus $\mathcal{A}$ and the Möbius strip $\mathcal{M}$. The last three amplitudes contain ultraviolet divergences which are conveniently analysed in the transverse channel. This channel describes the tree level exchange of zero momentum closed string modes between holes and/or crosscaps. The divergences appear there in the infrared limit and are associated with the exchange of tachyonic and massless modes. One ensures the tadpole condition, that is the cancellation of the divergences due to the massless modes in the total amplitude, by fixing the Chan-Paton group. Here, the divergence is related to the dilaton tadpole, a non-zero one point function of a closed vertex operator on the disk or on the projective plane. If the tadpole condition is not imposed, the low-energy effective action acquires a dilaton potential. Let us stress that in the present case the tadpole condition defining the bosonic open string is not compulsory: the presence of the dilaton tadpole does not render the theory inconsistent if the vacuum is shifted by the Fishler-Susskind mechanism [21].

The tadpole condition for the 26-dimensional uncompactified bosonic string determines the Chan-Paton group to be $S O\left(2^{13}\right)$. Let us discuss the derivation of this well-known result. Introducing a Chan-Paton multiplicity $n$ at both string ends, the four different one-loop amplitudes of the unoriented 26-dimensional bosonic string are given by (see for example 22]):

\footnotetext{
${ }^{12}$ In this paper, the elimination of massless tadpole divergences is always only imposed in leading order in the coupling constant.
} 


$$
\begin{array}{r}
\mathcal{T}=\int_{\mathcal{F}} \frac{d^{2} \tau}{\tau_{2}^{14}} \frac{1}{\eta^{24}(\tau) \bar{\eta}^{24}(\bar{\tau})}, \\
\mathcal{K}=\frac{1}{2} \int_{0}^{\infty} \frac{d \tau_{2}}{\tau_{2}^{14}} \frac{1}{\eta^{24}\left(2 i \tau_{2}\right)}, \\
\mathcal{A}=\frac{n^{2}}{2} \int_{0}^{\infty} \frac{d \tau_{2}}{\tau_{2}^{14}} \frac{1}{\eta^{24}\left(i \tau_{2} / 2\right)}, \\
\mathcal{M}=\frac{\epsilon n}{2} \int_{0}^{\infty} \frac{d \tau_{2}}{\tau_{2}^{14}} \frac{1}{\hat{\eta}^{24}\left(i \tau_{2} / 2+1 / 2\right)},
\end{array}
$$

where $\mathcal{F}$ is a fundamental domain of the modular group for the torus and $\eta(\tau)$ is the Dedekind function:

$$
\eta(\tau)=q^{\frac{1}{24}} \prod_{m=1}^{\infty}\left(1-q^{m}\right), \quad q=e^{2 \pi i \tau}, \quad \tau=\tau_{1}+i \tau_{2} .
$$

The 'hatted' Dedekind function in $\mathcal{M}$ means that the overall phase is dropped in $\eta\left(i \tau_{2} / 2+1 / 2\right)$ ensuring that $\hat{\eta}\left(i \tau_{2} / 2+1 / 2\right)$ is real. A similar notation will be used for a generic function $f$ admitting an expansion $f=q^{a} \sum_{i=0}^{\infty} a_{i} q^{i}$, namely $\hat{f}(\tau+1 / 2)=e^{-i \pi a} f(\tau+1 / 2)$. The world-sheet parity operator defining $\mathcal{M}$ is $\Omega=\epsilon(-1)^{N}$ where $N$ is the open string oscillator number operator. The plus or minus sign $\epsilon$ in Eq. (29) encodes the action of $\Omega$ on the vacuum and the one-half shift in the argument of the Dedekind function in Eq.(29) encodes the action of the twist operator $(-1)^{N}$.

The amplitudes $\mathcal{T} / 2+\mathcal{K}$ and $\mathcal{A}+\mathcal{M}$ are respectively the partition function of the closed and open unoriented string sectors. The $q$-independent term in the expansion of the integrand of $\mathcal{A}+\mathcal{M}$ gives the number of massless vectors and determines the nature of the Chan-Paton group. Using Eqs.(28) and (29) one finds $n(n-\epsilon) / 2$ massless vectors: if $\epsilon=+1$ (resp. $\epsilon=-1$ ), the Chan-Paton group is $S O(n)$ (resp. $U S p(n))$.

To impose the tadpole condition we interpret $\mathcal{K}, \mathcal{A}$ and $\mathcal{M}$ as amplitudes in the transverse (tree) channel. To this effect we first define $t=2 \tau_{2}$ (resp. $t=$ $\left.\tau_{2} / 2\right)$ in $\mathcal{K}$ (resp. $\mathcal{A}$ ) and express the modular form $\eta^{24}(i t)$ in the integrand of Eqs. (27) and (28) in terms of its $S$-transform. The change of variable $l=1 / t$ yields

$$
\mathcal{K}_{\text {tree }}=\frac{2^{13}}{2} \int_{0}^{\infty} d l \frac{1}{\eta^{24}(i l)}
$$




$$
\text { and } \quad \mathcal{A}_{\text {tree }}=\frac{n^{2} 2^{-13}}{2} \int_{0}^{\infty} d l \frac{1}{\eta^{24}(i l)} .
$$

The subscript tree emphasises that the expressions Eqs.(31) and (32), although identical to the integrals Eqs.(27) and (28), are now rewritten in terms of tree level intermediate states. Throughout the paper, any amplitude formulated in the 'direct' channel as a one loop amplitude $\mathcal{I}$, will be relabeled $\mathcal{I}_{\text {tree }}$ when expressed in terms of transverse channel tree level intermediate states.

It is a little bit more tricky to go from the direct to the transverse channel for the Möbius amplitude. One expresses $\hat{\eta}^{24}\left(i \tau_{2} / 2+1 / 2\right)$ in terms of its $P$-transform [10] which combines the modular transformations $S$ (i.e. $\tau \rightarrow$ $-1 / \tau)$ and $T$ (i.e. $\tau \rightarrow \tau+1)$ :

$$
P=T^{1 / 2} S T^{2} S T^{1 / 2} .
$$

One then performs the change of variable $l=1 /\left(2 \tau_{2}\right)$ to get

$$
\mathcal{M}_{\text {tree }}=2 \frac{\epsilon n}{2} \int_{0}^{\infty} d l \frac{1}{\hat{\eta}^{24}(i l+1 / 2)} .
$$

The tadpole condition can now be imposed by requiring the vanishing of the

$e^{-2 \pi l}$-independent term in the integrand of the total tree amplitude $\mathcal{K}_{\text {tree }}+$ $\mathcal{A}_{\text {tree }}+\mathcal{M}_{\text {tree }}$. One gets the following condition

$$
\left(2^{13}+2^{-13} n^{2}-2 \epsilon n\right)=2^{-13}\left(2^{13}-\epsilon n\right)^{2}=0,
$$

which singles out $\epsilon=+1$ and the value $n=2^{13}$.

Therefore, one recovers that the uncompactified open bosonic string theory obeying the tadpole condition is unoriented and has an $S O\left(2^{13}\right)$ Chan-Paton group 18 .

\subsection{Compactification of the open string ancestor on Lie algebra lattices and truncation}

We now explain how the open string theories in 10 dimensions may be obtained by truncation from the compactified 26-dimensional bosonic string. 
We begin by discussing the construction of open string descendants from closed strings compactified on the EN lattice of a semi-simple Lie group $\mathcal{G}$ of rank $d$. We specialise to the rank sixteen groups $\mathcal{G}=E_{8} \times E_{8}$ and $\mathcal{G}=E_{8} \times S O(16)$ and perform the truncation.

\subsubsection{Compactification on an EN lattice}

Consider the toroidal compactification of the bosonic closed string theory in 26 dimensions on the EN lattice of a semi-simple Lie group $\mathcal{G}$ of rank $d$.

The lattice partition function of the oriented closed string theory, whose gauge symmetry is $\mathcal{G} \times \mathcal{G}$, is

$$
\sum_{\beta=1}^{\mathcal{N}} \bar{\gamma}_{\beta, L}(\bar{\tau}) \gamma_{\beta, R}(\tau)
$$

where the summation extends over the $\mathcal{N}$ conjugacy classes of the lattice. $\mathcal{N}$ is equal to the order of the centre of the universal covering group of $\mathcal{G}$. We shall hereafter label the root lattice of $\mathcal{G}$ by $\beta=1$.

Unoriented closed strings are obtained by acting on the closed string states with the world-sheet parity projection operator $\left(1+\Omega_{c}\right) / 2$ where $\Omega_{c}$ interchanges the quantum numbers of the left and right sectors : $\Omega_{c}|L, R\rangle=$ $|R, L\rangle$. The Klein bottle amplitude $\mathcal{K}$ is obtained from the torus by inserting the operator $\Omega_{c} / 2$. From Eq.(36), we see that the lattice contribution to $\mathcal{K}$ is the sum over the $\mathcal{N}$ conjugacy class partition functions $\gamma_{\beta}$. Combining the lattice partition function with the partition function of the oscillator states in the non-compact dimensions, we get, instead of Eq.(27),

$$
\mathcal{K}=\frac{1}{2} \int_{0}^{\infty} \frac{d \tau_{2}}{\tau_{2}^{14-d / 2}} \frac{1}{\eta^{24-d}\left(2 i \tau_{2}\right)} \sum_{\beta=1}^{\mathcal{N}} \gamma_{\beta}\left(2 i \tau_{2}\right)
$$

Other choices for the lattice contribution to $\mathcal{K}$ are possible. They differ from the one in Eq.(37) by minus signs in the sum over the conjugacy classes in a way compatible with the fusion rules [10, 23]. They will not be considered here. 
To compute the transverse Klein bottle amplitude $\mathcal{K}_{\text {tree }}$, we need the Stransform of $\sum_{\beta=1}^{\mathcal{N}} \gamma_{\beta}(\tau)$. From Eq.(18) we get

$$
\sum_{\beta=1}^{\mathcal{N}} \gamma_{\beta}(\tau)=\sqrt{\mathcal{N}} \gamma_{1}\left(-\frac{1}{\tau}\right)
$$

Thus, from Eq.(37),

$$
\mathcal{K}_{\text {tree }}=\frac{2^{13}}{2} \frac{\sqrt{\mathcal{N}}}{2^{d / 2}} \int_{0}^{\infty} d l \frac{1}{\eta^{24-d}(i l)} \gamma_{1}(i l) .
$$

The torus and the Klein bottle amplitudes, which only have closed string intermediate states, are not altered by Chan-Paton multiplicities and we shall restrict ourselves to EN compactifications with Klein-bottle amplitude $\mathcal{K}$ given by Eq.(37). The corresponding $\mathcal{K}_{\text {tree }}$ is given by Eq.(39). For such $\mathcal{K}$, it will be possible to eliminate the dilaton tadpole.

We now turn to the open string sector.

The direct annulus amplitude generalising Eq.(28) in absence of Chan-Paton multiplicity only contains the root partition function $\gamma_{1}$ (see Appendix A). It is thus given by

$$
\mathcal{A}=\frac{1}{2} \int_{0}^{\infty} \frac{d \tau_{2}}{\tau_{2}^{14-d / 2}} \frac{1}{\eta^{24-d}\left(i \tau_{2} / 2\right)} \gamma_{1}\left(i \tau_{2} / 2\right) .
$$

The corresponding transverse amplitude, representing the exchange of closed string modes between two boundaries, follows from the $S$ transformation Eq.(38),

$$
\mathcal{A}_{\text {tree }}=\frac{2^{-13}}{2} \frac{2^{d / 2}}{\sqrt{\mathcal{N}}} \int_{0}^{\infty} d l \frac{1}{\eta^{24-d}(i l)} \sum_{\beta=1}^{\mathcal{N}} \gamma_{\beta}(i l)
$$

We now introduce Chan-Paton multiplicities. The transverse annulus amplitude is a tree amplitude with factorisable residues. Its most general form preserving the group symmetry is given by a sum on all conjugacy classes:

$$
\mathcal{A}_{\text {tree }}=\frac{2^{-13}}{2} \frac{2^{d / 2}}{\sqrt{\mathcal{N}}} \int_{0}^{\infty} d l \frac{1}{\eta^{24-d}(i l)} \sum_{\beta=1}^{\mathcal{N}}\left(a_{\beta}\right)^{2} \gamma_{\beta}(i l)
$$


with arbitrary coefficients $a_{\beta}$. We will see below that the normalisation chosen in Eq.(42) reduces to the normalisation in Eq.(41) in absence of ChanPaton multiplicities.

The direct annulus is obtained using the modular $S$-transform. Except in the particular case where all coefficients $\left(a_{\beta}\right)^{2}$ are equal, the direct amplitude contains in addition to the root partition function $\gamma_{1}$, contributions from other conjugacy classes. The latter differ from $\gamma_{1}$ by a shift $\mathbf{p}_{\beta}$ on the weight lattice of the compactified momenta. Therefore in general, Wilson lines must be introduced to accommodate this momentum shift in the open strings spectrum.

We make the following ansatz for $a_{\beta}$

$$
a_{\beta}=\sum_{\delta=1}^{\mathcal{N}} \exp \left(4 \pi i \alpha^{\prime} \mathbf{p}_{\beta} \cdot \mathbf{p}_{\delta}\right) n_{\delta}
$$

where the $n_{\delta}$ are positive integers or zero. Using Eq.(18), we get

$$
\frac{1}{\sqrt{\mathcal{N}}} \sum_{\beta=1}^{\mathcal{N}}\left(a_{\beta}\right)^{2} \gamma_{\beta}(i l)=\frac{1}{\mathcal{N}} \sum_{\beta, \delta, \sigma, \tau=1}^{\mathcal{N}} \exp \left[4 \pi i \alpha^{\prime} \mathbf{p}_{\beta} \cdot\left(\mathbf{p}_{\delta}+\mathbf{p}_{\sigma}+\mathbf{p}_{\tau}\right)\right] n_{\sigma} n_{\tau} \gamma_{\delta}\left(\frac{i}{l}\right) \text {. }
$$

The orthogonality theorem for characters of finite groups, as applied to the centre of the universal covering of $\mathcal{G}$, reads

$$
\sum_{\beta=1}^{\mathcal{N}} \exp \left(4 \pi i \alpha^{\prime} \mathbf{p}_{\beta} \cdot \mathbf{p}_{\sigma}\right) \exp \left(4 \pi i \alpha^{\prime} \mathbf{p}_{\beta} \cdot \mathbf{p}_{\tau}\right)=\mathcal{N} \delta_{\left(\mathbf{p}_{\sigma}+\mathbf{p}_{\tau}+\mathbf{p}\right)}
$$

where $\sqrt{2 \alpha^{\prime}} \mathbf{p}$ is an arbitrary vector of the root lattice. Eq.(45) applied to Eq.(44) gives

$$
\frac{1}{\sqrt{\mathcal{N}}} \sum_{\beta=1}^{\mathcal{N}}\left(a_{\beta}\right)^{2} \gamma_{\beta}(i l)=\sum_{\delta=1}^{\mathcal{N}} \sum_{\sigma, \tau=1}^{\mathcal{N}} n_{\sigma} n_{\tau} \gamma_{\delta}\left(\frac{i}{l}\right) \quad, \quad \mathbf{p}_{\sigma}+\mathbf{p}_{\tau}+\mathbf{p}_{\delta}+\mathbf{p}=0
$$

from which the direct amplitude $\mathcal{A}$ follows:

$$
\begin{array}{r}
\mathcal{A}=\frac{1}{2} \int_{0}^{\infty} \frac{d \tau_{2}}{\tau_{2}^{14-d / 2}} \frac{1}{\eta^{24-d}\left(i \tau_{2} / 2\right)} \sum_{\substack{\delta=1 \\
\mathbf{p}_{\sigma}+\mathbf{p}_{\tau}+\mathbf{p}_{\delta}+\mathbf{p}=0}}^{\mathcal{N}} n_{\sigma} n_{\tau} \gamma_{\delta}\left(i \tau_{2} / 2\right)
\end{array}
$$


Let us illustrate this result by applying it to $\mathcal{G}=S O(4 \mathrm{~m})$ (the characterisation of the conjugacy classes of $S O(4 \mathrm{~m})$ by shift vectors was given in Section 2.2). We get, using the constraint in Eq.(46),

$$
\begin{aligned}
\sum_{\delta, \sigma, \tau=1}^{\mathcal{N}} n_{\sigma} n_{\tau} \gamma_{\delta}\left(\frac{i}{l}\right)= & \left(n_{o}^{2}+n_{v}^{2}+n_{s}^{2}+n_{c}^{2}\right) \gamma_{(o)}\left(\frac{i}{l}\right)+\left(2 n_{o} n_{v}+2 n_{s} n_{c}\right) \gamma_{(v)}\left(\frac{i}{l}\right) \\
& +\left(2 n_{o} n_{s}+2 n_{s} n_{c}\right) \gamma_{(s)}\left(\frac{i}{l}\right)+\left(2 n_{o} n_{c}+2 n_{v} n_{s}\right) \gamma_{(c)}\left(\frac{i}{l}\right)
\end{aligned}
$$

Eq.(48) exemplifies two important features: first, the coefficient of the root partition function is a sum of squares; second, the coefficients in all other conjucacy class partition functions are sums of products $n_{\sigma} n_{\tau}, \sigma \neq \tau$. This indicates that $n_{o}, n_{v}, n_{s}, n_{c}$ label distinct Chan-Paton multiplicities.

Starting from the annulus amplitude $\mathcal{A}$ given in Eq.(47) we now construct the Möbius amplitude. The Möbius amplitude in the direct channel $\mathcal{M}$ only contains strings with identical Chan-Paton indices at both ends. If $\mathcal{A}$ exhibits the two features illustrated in the example Eq.(48), $\mathcal{M}$ only contains terms pertaining to the root lattice. Given the ansatz Eq.(43), such features arise if and only if $\mathbf{p}_{\alpha}+\mathbf{p}_{\beta}=\mathbf{p}$ for any $\alpha$ and $\beta$, as can be seen by comparing Eq.(46) with Eq.(48). Equivalently, they arise if and only if the centre of the covering group of $\mathcal{G}$ only contains elements of order less or equal to two.

We shall only consider hereafter EN compactifications on such groups. The simple simply laced Lie groups obeying this condition are listed in Table I and we restrict our considerations to such groups or direct products thereof.

\begin{tabular}{||c|c|c||c|c||}
\hline Group & Rank $(=d)$ & Center & $\sqrt{\mathcal{N}} / 2^{d / 2}$ & Rank of $b_{a b}$ \\
\hline$S U(2)$ & 1 & $Z_{2}$ & $2^{0}$ & 0 \\
\hline$S O(4 m) m>1$ & $2 m$ & $Z_{2} \times Z_{2}$ & $2^{1-m}$ & $2 m-2$ \\
\hline$E_{7}$ & 7 & $Z_{2}$ & $2^{-3}$ & 6 \\
\hline$E_{8}$ & 8 & 1 & $2^{-4}$ & 8 \\
\hline
\end{tabular}

Table I

We see that for all these groups, (and for their direct products), the factor $\sqrt{\mathcal{N}} / 2^{d / 2}$ entering Eq.(39) and (41) has the form $2^{-x}$ where $x$ is an integer 
greater than or equal to zero. The value $x=0$ is only realised at the Tduality self-dual point $[S U(2)]^{d}\left(=[S O(4)]^{d / 2}\right.$ for $d$ even $)$. In the last column, we relate the factor $\sqrt{\mathcal{N}} / 2^{d / 2}$ to the rank of the antisymmetric tensor $b_{a b}$. The latter will be used in Section $\mathbf{3 . 3}$ and is defined in Appendix A.

To get the explicit form of $\mathcal{M}$ we must use, instead of the standard twist $\Omega=\epsilon(-1)^{N}$, the group invariant operator ${ }^{13} \Omega_{\mathcal{G}}=\epsilon(-1)^{N+\alpha^{\prime} \mathbf{p}_{o p}^{2}}$ [13], where the eigenvalues of $\sqrt{2 \alpha^{\prime}} \mathbf{p}_{\text {op }}$ span the root lattice of $\mathcal{G}$. The additional term $\alpha^{\prime} \mathbf{p}_{o p}^{2}$ in the exponent is allowed because $\alpha^{\prime} \mathbf{p}^{2}$ is integer when $\sqrt{2 \alpha^{\prime}} \mathbf{p} \in \Lambda_{\text {root }}$. We obtain

$$
\mathcal{M}=\frac{\epsilon}{2} \int_{0}^{\infty} \frac{d \tau_{2}}{\tau_{2}^{14-d / 2}} \frac{1}{\hat{\eta}^{24-d}\left(i \tau_{2} / 2+1 / 2\right)}\left(\sum_{\sigma=1}^{\mathcal{N}} n_{\sigma}\right) \hat{\gamma}_{1}\left(i \tau_{2} / 2+1 / 2\right)
$$

which, using Eq.(43), can be expressed in terms of $a_{1}$ :

$$
a_{1}=\sum_{\sigma=1}^{\mathcal{N}} n_{\sigma}
$$

In Eq.(49), the twist $\Omega_{\mathcal{G}}$ is encoded by shifting both the argument of the Dedekind function and of the root lattice partition function by a half unit. Using the $P$-transform and changing variable to $l=1 /\left(2 \tau_{2}\right)$, we rewrite the Möbius amplitude in terms of closed strings propagating between hole and crosscap. For the groups $S O(8 \mathrm{~m})$ and $E_{8}$ we get

$$
\mathcal{M}_{\text {tree }}=2 \delta_{\mathcal{G}} \frac{\epsilon}{2} \int_{0}^{\infty} d l \frac{1}{\hat{\eta}^{24-d}(i l+1 / 2)}\left(\sum_{\sigma=1}^{\mathcal{N}} n_{\sigma}\right) \hat{\gamma}_{1}(i l+1 / 2),
$$

where $\delta_{\mathcal{G}}$ is a sign depending on the group $\mathcal{G}$ considered; $\delta_{\mathcal{G}}=+1$ for $E_{8}$ and $\delta_{\mathcal{G}}=(-1)^{m}$ for $S O(8 m)$. For $S U(2), S O(8 m+4)$ and $E_{7}$, the situation is different. The transverse amplitude $\mathcal{M}_{\text {tree }}$ derived from Eq. (49) is not proportional to $\hat{\gamma}_{1}$.

Two conditions have to be met for the consistency of the theory. First, $\mathcal{A}+\mathcal{M}$ must describe the partition function of unoriented open strings with

\footnotetext{
${ }^{13}$ The derivation of $\Omega_{\mathcal{G}}$ in the action formalism of Appendix $\mathbf{A}$ is given in Appendix $\mathbf{B}$.

${ }^{14}$ There are sign ambiguities in deriving $\mathcal{M}$ from $\mathcal{A}$ given by Eq.(47). We could a priori generalise $\epsilon \sum_{\sigma=1}^{\mathcal{N}} n_{\sigma}$ in Eq. (49) to $\sum_{\sigma=1}^{\mathcal{N}} \epsilon_{\sigma} n_{\sigma}$ with $\epsilon_{\sigma}= \pm 1$. This arbitrariness will be lifted by tree channel amplitudes where Eq.(50), which is true only if all $\epsilon_{\sigma}$ are equal, will be needed.
} 
products of Chan-Paton groups $S O(n)$ or $U S p(n)$ and second, each term in the $e^{-2 \pi l}$ power series expansion of the integrand of the total tree channel amplitude $\mathcal{K}_{\text {tree }}+\mathcal{A}_{\text {tree }}+\mathcal{M}_{\text {tree }}$ must be a perfect square.

With the ansatz Eq.(43), the first condition is always satisfied for compactification on EN lattices of the groups of Table I. The Chan-Paton groups are determined by the massless vector contributions to $\mathcal{A}+\mathcal{M}$, which appear as $q$-independent terms $\left(q=e^{-\pi \tau_{2} / 2}\right)$ in the expansion of the integrand of Eqs.(47) and (49). These $q$-independent terms are of two types: terms arising from level zero in $\eta$ and $\hat{\eta}$ and level one in $\gamma_{1}$ and $\hat{\gamma}_{1}$ are scalars (previously referred to as $\mathcal{G}$-scalars), while those arising from the level one in $\eta$ and $\hat{\eta}$ (which has degeneracy $(24-d)$ ) and level zero in $\gamma_{1}$ and $\hat{\gamma}_{1}$ are vectors. The number of such massless vectors is $\sum_{\sigma=1}^{\mathcal{N}} n_{\sigma}\left(n_{\sigma}-\epsilon\right) / 2$. We thus get $\mathcal{N}$ direct products of orthogonal (resp. symplectic) Chan-Paton groups for $\epsilon=+1$ (resp. $\epsilon=-1$ ) whose total multiplicity is $\sum_{\sigma=1}^{\mathcal{N}} n_{\sigma}$, provided the second condition is verified. This is the case for $E_{8}$ and $S O(8 \mathrm{~m})$ and we further analyse the unoriented bosonic theories compactified on these groups

These consistent theories also have two types of massless particles in the closed string channel: particles created by oscillators in non-compact dimensions and $\mathcal{G}$-scalars. Massless exchange generates divergences in the $e^{-2 \pi l}$ independent terms in the expansion of the integrand of $\mathcal{K}_{\text {tree }}+\mathcal{A}_{\text {tree }}+\mathcal{M}_{\text {tree }}$. There are massless exchanges in the terms proportional to $\gamma_{1}\left(\right.$ and $\hat{\gamma}_{1}$ ) in Eqs. (39), (42) and (51). The dilaton and the graviton are exchanged at level zero in $\gamma_{1}$ and level one in $\eta$ and massless $\mathcal{G}$-scalars are exchanged at level one in $\gamma_{1}$ and level zero in $\eta$. These divergences are all eliminated by imposing the dilaton tadpole condition:

$$
\frac{2^{13}}{2} \frac{\sqrt{\mathcal{N}}}{2^{d / 2}}+\frac{2^{-13}}{2} \frac{2^{d / 2}}{\sqrt{\mathcal{N}}} a_{1}^{2}-\delta_{\mathcal{G}} \epsilon a_{1}=\frac{2^{-13}}{2} \frac{2^{d / 2}}{\sqrt{\mathcal{N}}}\left(a_{1}-\delta_{\mathcal{G}} \epsilon 2^{13} \frac{\sqrt{\mathcal{N}}}{2^{d / 2}}\right)^{2}=0 .
$$

Choosing $\epsilon$ such that $\delta_{\mathcal{G}} \epsilon=+1$, we obtain from (50) the total Chan-Paton multiplicity :

$$
\sum_{\sigma=1}^{\mathcal{N}} n_{\sigma}=2^{13} \frac{\sqrt{\mathcal{N}}}{2^{d / 2}}
$$

In certain cases, there may be additional zero mass $\mathcal{G}$-scalars exchanges in $\mathcal{A}_{\text {tree }}$ in terms proportional to $\gamma_{\sigma}, \sigma \neq 1$. Imposing the tadpole condition for

\footnotetext{
${ }^{15}$ For the other groups listed in Table I, one may choose $\mathcal{K}$ different from Eq.(37) in order to fullfill the perfect square condition.
} 
these massless $\mathcal{G}$-scalars may lead to further restriction on the Chan-Paton groups, as will be seen in the next section.

The cases of interest for our analysis of truncation are $E_{8} \times E_{8}$ and $E_{8} \times$ $S O(16)$. As $\delta_{\mathcal{G}}=1$ for both groups, we must take $\epsilon=1$ to eliminate the dilaton tadpole. The Chan-Paton groups are

$$
\begin{aligned}
E_{8} \times E_{8} & \Rightarrow S O(n), \\
E_{8} \times S O(16) & \Rightarrow S O\left(n_{0}\right) \times S O\left(n_{v}\right) \times S O\left(n_{s}\right) \times S O\left(n_{c}\right),
\end{aligned}
$$

where $n, n_{0}, n_{v}, n_{s}, n_{c}$ are arbitrary positive integers or zero.

We now construct in detail the tadpole-free theories for EN compactification on these groups and perform the truncation.

\subsubsection{The $E_{8} \times E_{8}$ compactification and Type I superstring}

We consider here the compactification on the $E_{8} \times E_{8}$ lattice. In this case there is only one conjugacy class $(\mathcal{N}=1)$. We write

$$
\gamma_{1}=\gamma_{(o)_{E_{8}}} \gamma_{(o)_{E_{8}}}=\left(\gamma_{(o)_{16}}+\gamma_{(s)_{16}}\right)\left(\gamma_{(o)_{16}}+\gamma_{(s)_{16}}\right)
$$

The tree amplitudes given by Eqs.(39), (42) and (51), with $\delta_{\mathcal{G}}=\delta_{E_{8} \times E_{8}}=1$, read

$$
\begin{aligned}
\mathcal{K}_{\text {tree }} & =\frac{2^{5}}{2} \int_{0}^{\infty} d l \frac{1}{\eta^{8}(i l)} \gamma_{(o)_{E_{8}}}(i l) \gamma_{(o)_{E_{8}}}(i l) \\
\mathcal{A}_{\text {tree }} & =\frac{n^{2} 2^{-5}}{2} \int_{0}^{\infty} d l \frac{1}{\eta^{8}(i l)} \gamma_{(o)_{E_{8}}}(i l) \gamma_{(o)_{E_{8}}}(i l) \\
\mathcal{M}_{\text {tree }} & =\epsilon n \int_{0}^{\infty} d l \frac{1}{\hat{\eta}^{8}\left(i l+\frac{1}{2}\right)} \hat{\gamma}_{(o)_{E_{8}}}(i l+1 / 2) \hat{\gamma}_{(o)_{E_{8}}}(i l+1 / 2) .
\end{aligned}
$$

They can easily be expressed as direct channel amplitudes $\mathcal{K}, \mathcal{A}$ and $\mathcal{M}$.

We impose the vanishing of the dilaton tadpole. All the transverse amplitudes are proportional to the root lattice of $E_{8} \times E_{8}$. Therefore, as explained in the preceding section, all divergences are eliminated by the single constraint Eq.(53). This yields

$$
n=2^{5}
$$


and $\epsilon=+1$. The Chan-Paton group is given by Eq.(54). It is $S O(32)$. Let us stress again that this reduction of Chan-Paton group from $S O\left(2^{13}\right)$ by compactification is crucially related to the structure of the Lie algebra lattice, and in particular to the number of conjugacy classes, as shown in Eq. (53).

We are now in position to derive the truncated theory from the tadpole-free bosonic open string theory compactified on the EN lattice of $E_{8} \times E_{8}$.

Eq.(13) gives

$$
\begin{gathered}
\gamma_{(o)_{E 8}} \gamma_{(o)_{E 8}}=\gamma_{(o)_{E 8}}\left(\gamma_{(o)_{16}}+\gamma_{(s)_{16}}\right) \\
\text { Trunc } \downarrow \text { Flip } \\
\gamma_{(v)_{8}}-\gamma_{(s)_{8}}
\end{gathered}
$$

and the transverse amplitudes Eqs.(57), (58) and (59) become after truncation

$$
\begin{aligned}
\mathcal{K}_{\text {tree }}^{t} & =\mathcal{A}_{\text {tree }}^{t}=\frac{2^{5}}{2} \int_{0}^{\infty} d l \frac{1}{\eta^{8}(i l)}\left(\gamma_{(v)_{8}}-\gamma_{(s)_{8}}\right)(i l) \\
\mathcal{M}_{\text {tree }}^{t} & =-2^{5} \int_{0}^{\infty} d l \frac{1}{\hat{\eta}^{8}(i l+1 / 2)}\left(\hat{\gamma}_{(v)_{8}}-\hat{\gamma}_{\left.(s)_{8}\right)}\right)(i l+1 / 2) .
\end{aligned}
$$

The flip in sign in Eq.(62) as compared to Eq.(59) with $\epsilon=+1$ is solely due to the definition of hatted functions. Truncating the direct amplitudes $\mathcal{A}$ and $\mathcal{M}$ we get

$$
\begin{aligned}
\mathcal{A}^{t} & =\frac{2^{10}}{2} \int_{0}^{\infty} \frac{d \tau_{2}}{\tau_{2}^{6}} \frac{1}{\eta^{8}\left(i \tau_{2} / 2\right)}\left(\gamma_{(v)_{8}}-\gamma_{(s)_{8}}\right)\left(i \tau_{2} / 2\right) \\
\mathcal{M}^{t} & =-\frac{2^{5}}{2} \int_{0}^{\infty} \frac{d \tau_{2}}{\tau_{2}^{6}} \frac{1}{\hat{\eta}^{8}\left(i \tau_{2} / 2+1 / 2\right)}\left(\hat{\gamma}_{(v)_{8}}-\hat{\gamma}_{\left.(s)_{8}\right)}\right)\left(i \tau_{2} / 2+1 / 2\right)
\end{aligned}
$$

The amplitudes in Eqs. 63) and (64) are equal to those in Eqs. 61) and (62) expressed in the transverse channel, a consequence of the fact that the truncation-flip commutes with $S$ and $T$ transformations.

Massless modes in both open and closed string channels, created by bosonic oscillators in non compact dimensions, become massive after truncation. A 
subset of massless $\mathcal{G}$-scalars are transmuted to massless modes. In the closed string channel, these are massless spinors, $N S-N S$ and $R$ - $R$ fields. The $N S$ $N S$ and $R$ - $R$ tadpoles are eliminated by the condition Eq.(60) inherited from the bosonic string. This is easily checked from Eqs.(61) and (62) describing the truncated tree amplitude $\mathcal{K}_{\text {tree }}^{t}+\mathcal{A}_{\text {tree }}^{t}+\mathcal{M}_{\text {tree }}^{t}$. Note that the flip in the $\epsilon$ sign in Eq.(62) is in accordance with $\delta_{\mathcal{G}} \epsilon=+1$, as $\delta_{S O(8)}=-1$. This flip in sign translates the fact that the zero mass states which contribute to the truncated tree amplitudes Eqs. (61) and (62) stem from the lattice partition functions at level zero, while the $\mathcal{G}$-scalars in the transverse amplitudes of the untruncated bosonic theory given in Eqs.(57), (58) and (59) arose at level one.

The Chan-Paton group $S O(32)$ is preserved under truncation. This can be checked by counting, in the open channel, the number of massless vectors arising by truncation from $\mathcal{G}$-scalars. This number (which is the same as the number of massless spinors) is $2^{5}\left(2^{5}-1\right) / 2$, as is immediately apparent from the partition function $\mathcal{A}^{t}+\mathcal{M}^{t}$ given by Eqs.(63) and (64). The latter is then the partition function of the open string sector of Type I. Note that the $2^{5}\left(2^{5}-1\right) / 2$ vectors of the untruncated theory stem from the Dedekind function at level one in $\mathcal{A}+\mathcal{M}$ while the vectors of the truncated theory stem from level zero in $\mathcal{A}^{t}+\mathcal{M}^{t}$, a manifestation of the change in the Lorentz group in truncation which allows the transmutation of $\mathcal{G}$-scalars to tensors. Similar remarks to those concerning the flip of $\epsilon$ sign in the closed string channel can be made in the open channel.

We see that the truncation of the unoriented tadpole-free bosonic open string theory, compactified on the $E_{8} \times E_{8}$ lattice, results in the $S O(32)$ anomaly free Type I theory.

\subsubsection{The $E_{8} \times S O(16)$ compactification and Type 0 strings}

We now consider the 26-dimensional unoriented bosonic open string theory compactified on the $E_{8} \times S O(16)$ lattice. In this case there are four conjugacy classes, $\mathcal{N}=4: \gamma_{(o)_{E_{8}}} \gamma_{(o)_{16}}, \gamma_{(o)_{E_{8}}} \gamma_{(v)_{16}}, \gamma_{(o)_{E_{8}}} \gamma_{(s)_{16}}, \gamma_{(o)_{E_{8}}} \gamma_{(c)_{16}}$ and we have

$$
\gamma_{1}=\gamma_{(o)_{E_{8}}} \gamma_{(o)_{16}} \text {. }
$$


The tree amplitudes given by Eqs.(39), (42), and (51), with $\delta_{E_{8} \times S O(16)}=1$, read

$$
\begin{aligned}
\mathcal{K}_{\text {tree }} & =\frac{2^{6}}{2} \int_{0}^{\infty} d l \frac{1}{\eta^{8}} \gamma_{(o)_{16}} \gamma_{(o)_{E 8}} \\
\mathcal{A}_{\text {tree }} & =\frac{2^{-6}}{2} \int_{0}^{\infty} d l \frac{1}{\eta^{8}}\left(a_{1}^{2} \gamma_{(o)_{16}}+a_{2}^{2} \gamma_{(v)_{16}}+a_{3}^{2} \gamma_{(s)_{16}}+a_{4}^{2} \gamma_{(c)_{16}}\right) \gamma_{(o)_{E_{8}}},(67) \\
\mathcal{M}_{\text {tree }} & =\epsilon a_{1} \int_{0}^{\infty} d l \frac{1}{\hat{\eta}^{8}} \hat{\gamma}_{(o)_{16}} \hat{\gamma}_{(o)_{E_{8}}}
\end{aligned}
$$

and

$$
\begin{array}{ll}
a_{1}=n_{o}+n_{v}+n_{s}+n_{c}, & a_{2}=n_{o}+n_{v}-n_{s}-n_{c}, \\
a_{3}=n_{o}-n_{v}+n_{s}-n_{c}, & a_{4}=n_{o}-n_{v}-n_{s}+n_{c} .
\end{array}
$$

We enforce the tadpole conditions. In this case there are four tadpole conditions because there are four different types of massless modes giving rise to divergent contributions in the tree amplitudes: in addition to the graviton and dilaton encoded in the Dedekind function at level one, there are three types of $\mathcal{G}$-scalars depicted respectively by $\gamma_{(o)_{16}}$ at level 1 and $\gamma_{(s)_{16}}, \gamma_{(c)_{16}}$ at level zero. The tadpole conditions which eliminate the divergences at level one in the Dedekind function and in $\gamma_{(o)_{16}}$ are both given by Eq.(53):

$$
n_{o}+n_{v}+n_{s}+n_{c}=a_{1}=64
$$

with $\epsilon=1$. The Chan-Paton group is given by Eq.(55). It is $S O\left(n_{o}\right) \times$ $S O\left(n_{v}\right) \times S O\left(n_{s}\right) \times S O\left(n_{c}\right)$, with $n_{o}+n_{v}+n_{s}+n_{c}=64$. The pattern of symmetry breaking of $S O(64)$ is further determined by imposing the two remaining tadpole conditions corresponding to the $\gamma_{(s)_{16}}$ and $\gamma_{(c)_{16}}$ divergences.. They give:

$$
\begin{array}{ccc}
a_{3}=0 \quad, \quad a_{4}=0 \\
\\
n_{o}=n_{v} \quad, \quad n_{s}=n_{c}
\end{array}
$$

The Chan-Paton group of the tadpole-free unoriented bosonic open string theory compactified on the $E_{8} \times S O(16)$ lattice is thus:

$$
S O(n) \times S O(n) \times S O(32-n) \times S O(32-n) .
$$


We now derive the truncated theory.

Using Eq.(13), the truncation-flip

$$
\begin{array}{ll}
\gamma_{(o)_{16}} \rightarrow+\gamma_{(v)_{8}}, & \gamma_{(v)_{16}} \rightarrow+\gamma_{(o)_{8}}, \\
\gamma_{(s)_{16}} \rightarrow-\gamma_{(s)_{8}}, & \gamma_{(c)_{16}} \rightarrow-\gamma_{(c)_{8}},
\end{array}
$$

applied to the amplitudes Eqs.(66), (67) and (68) gives,

$$
\begin{aligned}
\mathcal{K}_{\text {tree }}^{t} & =\frac{2^{6}}{2} \int_{0}^{\infty} d l \frac{1}{\eta^{8}} \gamma_{(v)_{8}}, \\
\mathcal{A}_{\text {tree }}^{t} & =\frac{2^{-6}}{2} \int_{0}^{\infty} d l \frac{1}{\eta^{8}}\left[(64)^{2} \gamma_{(v)_{8}}+16(n-16)^{2} \gamma_{(o)_{8}}\right], \\
\mathcal{M}_{\text {tree }}^{t} & =-64 \int_{0}^{\infty} d l \frac{1}{\hat{\eta}^{8}} \hat{\gamma}_{(v)_{8}} .
\end{aligned}
$$

The transverse amplitudes propagate massless $N S-N S$ particles at level zero in $\gamma_{(v)_{8}}$, inherited from $\mathcal{G}$-scalars propagating in $\gamma_{(o)_{16}}$. The potential $N S$-NS tadpole has been eliminated by the condition Eq.(70). The truncated direct amplitudes $\mathcal{A}^{t}$ and $\mathcal{M}^{t}$ follow from Eq.(74) and (75) using $S$ and $P$ transformations or equivalently by truncating the direct amplitudes. These now contain all four conjugacy classes of $S O(8)$ but $\mathcal{M}$ only contains $\hat{\gamma}_{(v)_{8}}$. This indicates that open strings with identical Chan-Paton factors at both ends only have the bosonic $N S_{+}$spectrum with the tachyon projected out, while open strings with distinct Chan-Paton factors at their ends have tachyons or fermionic states of either chirality.

As in the preceding section, the massless states in both open and closed channels again arise from a subset of $\mathcal{G}$-scalars and the truncation exhibits the concomitant flip of $\epsilon$ sign between $\mathcal{M}_{\text {tree }}$ in Eq.(68) and $\mathcal{M}_{\text {tree }}^{t}$ in Eq.(75). The Chan-Paton group is transferred from the untruncated bosonic theory to the truncated fermionic theory and we obtain the spectrum of the $[S O(32-$ $n) \times S O(n)]^{2}$ Type O theories discussed in ref. [10, 24].

We see that the truncation of the unoriented tadpole-free bosonic open string theory, compactified on the $E_{8} \times S O(16)$ lattice, results in the tadpole-free 10-dimensional Type $\mathrm{O}$ theory with gauge group $[S O(32-n) \times S O(n)]^{2}$. Note that the Chan-Paton group has a higher rank than in Type I because $E_{8} \times S O(16)$ has more conjugacy classes than $E_{8} \times E_{8}$. We emphasise that 
this Chan-Paton gauge structure and the symmetry breaking pattern arise entirely from bosonic considerations.

\subsection{Brane fusion}

In this section we discuss the interpretation of the compactified $S O\left(2^{13}\right)$ unoriented bosonic theory in terms of D-branes and orientifolds.

The uncompactified $S O\left(2^{13}\right)$ bosonic string has a clear geometrical interpretation [25]. The ends of the open strings live on D25-branes and the tension of a D25-brane can be derived from $\mathcal{A}_{\text {tree }}$ (see Eq.(32) with $n=1$ ) by comparing with the field theory calculation [25].

The tension $T_{D 25}^{\text {bosonic }}$ is given by

$$
T_{D 25}^{\text {bosonic }}=\frac{\sqrt{\pi}}{2^{4} \kappa_{26}}\left(2 \pi \alpha^{1 / 2}\right)^{-14},
$$

where $\kappa_{26}^{2}=8 \pi G_{26}$ and $G_{26}$ is the Newtonian constant in 26 dimensions.

The action of the world-sheet parity operator on the 26-dimensional closed bosonic string introduces an orientifold 25-plane O25. The tension of the O25 can be derived from $\mathcal{K}_{\text {tree }}$ (and $\mathcal{M}_{\text {tree }}$ for the sign of this tension) again by comparing with the field theory calculation. The result is [25]:

$$
T_{O 25}^{\text {bosonic }}=-2^{12} T_{D 25}^{\text {bosonic }} .
$$

Therefore the tadpole condition fixing the $S O\left(2^{13}\right)$ gauge group means in this context that one has to introduce $n=2^{13}$ D25-branes ( $2^{12}+$ their images) to cancel the negative tension of the $O 25$ [25].

We now derive the tension of the wrapped orientifold and D25-branes for compactifications on EN lattices with Lie group $\mathcal{G}$ of rank $d$, where $\mathcal{G}$ is a direct product of the groups listed in table I. To this effect we describe the lattice in terms of constant background metric $g_{a b}$ and Neveu-Schwarz antisymmetric tensor $b_{a b}$. For EN lattices, these are quantised and their explicit form is given in appendix $\mathbf{A}$.

\footnotetext{
${ }^{16}$ We always give the Dp-brane tensions computed in the oriented closed theory.
} 
The squared wrapped orientifold tension is obtained from the coupling to gravity in the transverse Klein bottle amplitude $\mathcal{K}_{\text {tree }}$. For the groups $E_{8}$ and $S O(8 \mathrm{~m})$ and their direct products, $\mathcal{K}_{\text {tree }}$ contains dilaton and graviton exchange terms at level zero in $\gamma_{1}$, as exhibited in Eq.(39). We recall that $\mathcal{K}_{\text {tree }}$ is obtained from the direct amplitude $\mathcal{K}$ by the $\mathrm{S}$ modular transformation, and that $\mathcal{K}$ follows from the torus amplitude by inserting the world-sheet parity operator $\Omega_{c}$ which interchanges left and right sectors. This projects out the $b_{a b}$-field as can be checked by imposing $\mathbf{p}_{\mathbf{L}}=\mathbf{p}_{\mathbf{R}}$ in Eq.(A.11). For the above groups, the tension of the wrapped orientifold $T_{O 25}^{b o s o n i c}$ after compactification is then given, up to a sign, by:

$$
T_{O 25 \text { wr }}^{\text {bosonic }}=T_{O 25}^{\text {bosonic }}\left[(2 \pi)^{d} \sqrt{g}\right],
$$

where $g$ is the determinant of the metric. This can be verified by comparing the zero mass propagation in Eq.(39) with its field-theoric counterpart.

On the other hand the world-volume action of a D25-brane is given by the Born-Infeld action [26, 27, 19]. Accordingly, after compactification, the tension $T_{D 25}^{\text {bosonic }}$ of a wrapped D25-brane is given by 28:

$$
T_{D 25 \text { wr }}^{\text {bosonic }}=T_{D 25}^{\text {bosonic }}\left[(2 \pi)^{d} \sqrt{e}\right]=n_{f} T_{D 25}^{\text {bosonic }}\left[(2 \pi)^{d} \sqrt{g}\right],
$$

where $e$ is the determinant of $e_{a b} \equiv g_{a b}+b_{a b}$, and

$$
n_{f}=\sqrt{e / g} .
$$

The choice $\delta_{\mathcal{G}} \epsilon=+1$ in the transverse Möbius amplitude Eq.(51), which allows the cancellation of the dilaton tadpole divergence, means that the tension of the wrapped orientifold Eq. (78) is negative. This tension can then be compensated by introducing $n_{w}$ wrapped D25 branes (including images) with:

$$
n_{w}=2^{13} \sqrt{\frac{g}{e}}=\frac{2^{13}}{n_{f}} .
$$

The reduction factor $n_{f}$ defined in Eq. (80) is computed in Appendix C. One gets

$$
n_{f}=\frac{2^{d / 2}}{\sqrt{\mathcal{N}}}
$$

Inserting this value in Eq.(81), we recover the dilaton tadpole condition Eq.(53), with the total Chan-Paton multiplicity equal to the number $n_{w}$ 
of wrapped D-branes required for cancelling the wrapped orientifold tension. In other words, when the tadpole condition is imposed, the reduction factor $n_{f}$ coincides with the ratio of Chan-Paton multiplicities before and after compactification.

Recall that for the Lie groups $\mathcal{G}$ listed in table I and direct products thereof, the factor $2^{d / 2} / \sqrt{\mathcal{N}}=n_{f}$ has the form $2^{q}$, with $q$ a positive integer or zero. For compactifications on EN lattices, the integer $q$ is related to the rank $r$ of the $b_{a b}$ matrix listed in Table I. We have indeed

$$
\frac{2^{d / 2}}{\sqrt{\mathcal{N}}}=2^{r / 2}
$$

as can be verified by direct inspection from the explicit form of $b_{a b}$ given in Appendix A.

One may interpret the reduction of the total Chan-Paton multiplicity as a consequence of 'brane fusion'. Eq.(79) shows that one D25-brane of the 26-dimensional bosonic string theory, compactified on EN lattices with $\mathcal{G}$ in Table I, has the same tension as the integer number $n_{f}=\sqrt{e / g}$ of D25-branes when the theory is compactified on a generic Cartesian torus (for which $\left.b_{a b}=0\right)$ with equal volume. This fact does not depend on the orientability of the string nor on the tadpole condition. In presence of the enhanced symmetry, the rôle of the quantised $b_{a b}$-field is to fuse $n_{f}$ component branes into one. If the tadpole condition is imposed for groups with factors $E_{8}$ and $S O(8 m)$, one gets the concomitant reduction in the Chan-Paton multiplicity from the unoriented 26-dimensional bosonic string theory with Chan-Paton group $S O\left(2^{13}\right)$. Such reduction, and its expression in terms of the rank of the $b_{a b}$ matrix, is in agreement with reference [11].

We now specialise to $d=16$ and apply these results to $E_{8} \times E_{8}$ and $E_{8} \times$ $S O(16)$.

For $E_{8} \times E_{8}$ we have $\mathcal{N}=1$ and Eq.(82) gives $n_{f}=2^{8}$. Consequently $2^{8}$ D25-branes $\rightarrow$ one D25-brane and we are left with $2^{5}$ wrapped D25-branes. This is consistent with the fact that the Chan-Paton group of the tadpole-free $E_{8} \times E_{8}$ compactification is $S O(32)$.

For $E_{8} \times S O(16)$ we have $\mathcal{N}=4$. Thus Eq. (82) gives $n_{f}=2^{7}$. Consequently $2^{7}$ D25-branes $\rightarrow$ one D25-brane and we are left with $2^{6}$ wrapped D25-branes. 
This is consistent with the fact that the Chan-Paton group of the tadpole-free $E_{8} \times S O(16)$ compactification is $[S O(32-n) \times S O(n)]^{2}$.

\subsection{Energy conservation in the truncation}

We now show that truncation conserves energy. Namely the orientifold and D-brane tensions after truncation are equal to the ten-dimensional orientifold and D-brane tensions of the compactified bosonic ancestor.

We first discuss the orientifold tensions. Before truncation, the square of the 10-dimensional tension of the bosonic wrapped orientifold is proportional to the $q^{0}$-term $\left(q=e^{-2 \pi l}\right)$ in the integrand of $\mathcal{K}_{\text {tree }}$ due to the exchange of massless space-time modes. Using Eqs. (57) and (66), we have for both $E_{8} \times E_{8}$ and $E_{8} \times S O(16):$

$$
\begin{aligned}
& \left.\mathcal{K}_{\text {tree }}\right|_{q^{0}}=\left.\left.2^{4} \sqrt{\mathcal{N}} \quad \eta^{-8}(i l)\right|_{\text {level } 1} \gamma_{1}(i l)\right|_{\text {level } 0} \\
& =2^{4} \sqrt{\mathcal{N}} q^{-8 / 24}(8 q) \quad q^{-16 / 24}=2^{4} \sqrt{\mathcal{N}} 8 .
\end{aligned}
$$

After truncation the square of orientifold tension is proportional to the $q^{0}$ term in the integrand of $\mathcal{K}_{\text {tree }}^{t}$ coming from $\gamma_{(v)}$. Using Eqs. (61) and (73), we have for both $E_{8} \times E_{8}$ and $E_{8} \times S O(16)$

$$
\begin{aligned}
\mathcal{K}_{\text {tree }\left.\right|_{q^{0}}}^{t} & \left.\left.2^{4} \sqrt{\mathcal{N}} \quad \eta^{-8}(i l)\right|_{\text {level } 0} \gamma_{(v)_{8}}(i l)\right|_{\text {level } 0} \\
& =2^{4} \sqrt{\mathcal{N}} \quad q^{-8 / 24} \quad\left[q^{-4 / 24}\left(8 q^{+1 / 2}\right)\right]=2^{4} \sqrt{\mathcal{N}} 8
\end{aligned}
$$

The amplitudes Eq.(84) and Eq.(85) are independent of coupling constants. Their equality implies therefore that the orientifold tension $T_{09}^{f e r m}$ generated by truncation is equal to the tension of the wrapped bosonic orientifold $T_{O 25}^{\text {bosonic }}$ wh when expressed in terms of the same 10-dimensional Newton constant $\kappa_{10}$ :

$$
\kappa_{10}=\kappa_{26}\left((2 \pi)^{16} \sqrt{g}\right)^{-1 / 2}=\kappa_{26}\left(2 \pi \alpha^{1 / 2}\right)^{-8} 2^{4} \mathcal{N}^{-1 / 4} .
$$

The conservation of the orientifold tension in the truncation process implies in turns that the 10-dimensional tension of the D-branes is conserved. Indeed the truncated theories being tadpole-free, the total tension of the $n_{w}$ D9-branes generated by truncation from the compactified theory with $n_{w}$ wrapped D25-branes must cancel the orientifold tension. Using Eqs.(86), 
(76), (79) and (C.31) the tension $T_{D 9}^{\text {ferm }}$ of D9-branes in the truncated theory is thus predicted to be

$$
T_{D 9}^{f e r m}=T_{D 25 w r}^{\text {bosonic }}=\frac{\sqrt{\pi}}{2^{4} \kappa_{26}}\left(2 \pi \alpha^{\prime 1 / 2}\right)^{2}=\frac{\sqrt{\pi}}{\kappa_{10}}\left(2 \pi \alpha^{1 / 2}\right)^{-6} \mathcal{N}^{-1 / 4} .
$$

For $E_{8} \times E_{8}, \mathcal{N}=1$ and Eq. 87 gives

$$
T_{D 9}^{f e r m}=\frac{\sqrt{\pi}}{\kappa_{10}}\left(2 \pi \alpha^{\prime 1 / 2}\right)^{-6},
$$

which is indeed the tension of the Type IIB D9-brane (see [25]).

For $E_{8} \times S O(16), \mathcal{N}=4$ and Eq.(87) gives

$$
T_{D 9}^{f e r m}=\frac{\sqrt{\pi}}{\sqrt{2} \kappa_{10}}\left(2 \pi \alpha^{1 / 2}\right)^{-6},
$$

which is indeed the tension of the Type OB D9-branes (see for instance [29]).

\section{Discussions}

Although truncation is a universal procedure to extract all ten-dimensional closed fermionic string theories out of the closed bosonic string, one might have the feeling that such a procedure is essentially kinematical in character. One may always bosonise the world-sheet fermions to obtain, in the lightcone gauge, theories formally identical to those resulting from truncation $\square$. The added information provided by truncation is the encoding of all these theories in the 26-dimensional bosonic string theory compactified, in the left and/or right sector, on the Lie algebra lattice of $E_{8} \times S O(16)$. In particular, truncation selects the supersymmetric strings by extracting the only sublattice of $E_{8} \times S O(16)$ (namely $E_{8} \times E_{8}$ ) which yields a distinct compactification of the bosonic string consistent with modular invariance. This emergence of all fermionic strings from subspaces of the bosonic string Hilbert space in a systematic way is certainly impressive, but it is unclear that truncation can predict specific properties of the fermionic strings.

\footnotetext{
${ }^{17}$ See 16 and references therein.
} 
As a first step to convince oneself that truncation hides some dynamics, one would like to find such predictions.

This was the motivation which led us to generalise truncation to open strings. There, crucial ingredients of the fermionic strings, such as the cancellation of anomalies in Type I theory by the Chan-Paton group $S O(32)$, a priori seemed to bear no relation with the bosonic theory. Remarkably however, through the brane fusion mechanism, the imposition of a tadpole condition in the bosonic string explains this anomaly cancellation. It also yields the Chan-Paton groups which eliminate all tadpoles in Type $\mathrm{O}$ theories. In addition, the tensions of the fermionic space-filling D-branes are computed using properties of the bosonic string only. More important even is the fact that these results follow from specific properties of the $E_{8} \times S O(16)$ lattice, singling out this lattice out of all possible toroidal compactifications.

We think that this host of results points towards a dynamical origin of truncation. But such dynamics cannot be handled in the context of the perturbative approach given here. To settle the issue, a manageable non-perturbative approach to the bosonic string is mandatory. An attempt towards formulating such an approach in a classical limit has been recently proposed 30 and the present analysis suggests that such efforts should be further pursued.

If truncation is indeed a dynamical phenomenon, the central rôle played by the 26-dimensional bosonic string theory as a parent of all the fermionic strings would have important consequences for quantum gravity. Whatever may ultimately happen to string theory itself, it would probably mean that the fundamental constituents of quantum gravity do not contain fermionic degrees of freedom. 


\section{Acknowledgments}

François Englert is grateful to Peter West for a fruitful conversation. Laurent Houart thanks Augusto Sagnotti for illuminating discussions. We thank Auttakit Chattaraputi and Alexander Sevrin for constructive comments. F.E. acknowledges EPSRC for a Visiting Fellowship GR/N22793/01. A.T. acknowledges the Leverhulme Trust for a fellowship. This work is supported by the European Commission RTN program HPRN-CT-2000-00131 in which

F.E. is associated to the University of Brussels (ULB) and L.H. is associated to the University of Milano Bicocca (Milano 2). 


\section{A Toroidal compactification on EN lattices}

Toroidal compactification of the bosonic string is described by constant metric and antisymmetric tensors. The action is [12

$$
\begin{array}{r}
S=\frac{-1}{4 \pi \alpha^{\prime}} \int d \sigma d \tau\left[\left\{g_{a b} \partial_{\alpha} X^{a} \partial^{\alpha} X^{b}+\right.\right. \\
\left.b_{a b} \epsilon^{\alpha \beta} \partial_{\alpha} X^{a} \partial_{\beta} X^{b}\right\} \\
\left.+\eta_{\mu \nu} \partial_{\alpha} X^{\mu} \partial^{\alpha} X^{\nu}\right]
\end{array}
$$

with $g_{a b}, b_{a b}$ a constant metric and antisymmetric tensor in compact directions $(a, b=1, \ldots d)$,

$\eta_{\mu \nu}, \eta_{\alpha \beta}=(-1 ;+1, \ldots), \quad \mu, \nu=1, \ldots, s+2, \quad \alpha, \beta=1,2$,

$\epsilon^{01}=\epsilon^{\tau \sigma}=+1$,

$0 \leq \sigma \leq \pi$.

The fields $X^{a}$ are dimensionless and periodic in the target space with period $2 \pi ; g_{a b}$ and $b_{a b}$ have dimension $[L]^{2}$. Their conjugate momenta are

$$
\Pi_{a}(\sigma, \tau)=\frac{1}{2 \pi \alpha^{\prime}}\left(g_{a b} \partial_{\tau} X^{b}-b_{a b} \partial_{\sigma} X^{b}\right) .
$$

The configuration space torus is defined by a periodic lattice

$$
\mathbf{x} \equiv \mathbf{x}+2 \pi \mathbf{L}
$$

where $\mathbf{L}$ can be decomposed in the basis $\left\{\mathbf{e}_{\mathbf{a}}\right\}$

$$
\mathbf{L}=n^{a} \mathbf{e}_{\mathbf{a}} \quad, \quad n^{a} \in Z .
$$

The metric of the lattice is given by

$$
g_{a b}=\mathbf{e}_{\mathbf{a}} \cdot \mathbf{e}_{\mathbf{b}} .
$$

The metric and antisymmetric tensors which give rise to an EN lattice in the closed string sector with gauge group $\mathcal{G} \times \mathcal{G}$ are characterised by [31]

$$
\mathbf{e}_{\mathbf{a}}=\frac{1}{2} \sqrt{2 \alpha^{\prime}} \mathbf{r}_{\mathbf{a}}
$$

where the vectors $\mathbf{r}_{\mathbf{a}}$ form a basis of the root lattice $\Lambda_{\text {root }}$ of $\mathcal{G}$ and are chosen to be the simple roots with $\mathbf{r}_{\mathbf{a}} \cdot \mathbf{r}_{\mathbf{a}}=2$. The constant antisymmetric background field is 31

$$
b_{a b}=+\mathbf{e}_{\mathbf{a}} \cdot \mathbf{e}_{\mathbf{b}} \text { for } a>b,=-\mathbf{e}_{\mathbf{a}} \cdot \mathbf{e}_{\mathbf{b}} \text { for } a<b ; \quad b_{a b}=0 \text { for } a=b .
$$


It follows from these equations that $\left(2 / \alpha^{\prime}\right) g_{a b}$ is the Cartan matrix of $\mathcal{G}$ and therefore,

$$
\frac{2}{\alpha^{\prime}} g_{a b} \in Z \text {. }
$$

We also have

$$
\frac{2}{\alpha^{\prime}} b_{a b} \in Z
$$

The dual basis $\mathbf{e}^{\mathbf{a}}$ of $\mathbf{e}_{\mathbf{a}}$ is

$$
\mathbf{e}^{\mathbf{a}}=2\left(2 \alpha^{\prime}\right)^{-1 / 2} \mathbf{w}^{\mathbf{a}}
$$

where the $\mathbf{w}^{\mathbf{a}}$ are the fundamental weights. The left and right momenta are given by:

$$
\begin{aligned}
& \mathbf{p}_{\mathbf{R}}=\left[\left(\frac{1}{2} m_{a}-b_{a b} n^{b}\left(2 \alpha^{\prime}\right)^{-1}\right) \mathbf{e}^{\mathbf{a}}+n^{a}\left(2 \alpha^{\prime}\right)^{-1} \mathbf{e}_{\mathbf{a}}\right] \\
& \mathbf{p}_{\mathbf{L}}=\left[\left(\frac{1}{2} m_{a}-b_{a b} n^{b}\left(2 \alpha^{\prime}\right)^{-1}\right) \mathbf{e}^{\mathbf{a}}-n^{a}\left(2 \alpha^{\prime}\right)^{-1} \mathbf{e}_{\mathbf{a}}\right] .
\end{aligned}
$$

One verifies that

- a) the left and right momenta $\sqrt{2 \alpha^{\prime}} \mathbf{p}_{\mathbf{R}}$ and $\sqrt{2 \alpha^{\prime}} \mathbf{p}_{\mathbf{L}}$ lie on the weight lattice $\Lambda_{\text {weight }}$ of $\mathcal{G}$

- b) the momenta $\sqrt{2 \alpha^{\prime}} \mathbf{p}_{\mathbf{R}}$ and $\sqrt{2 \alpha^{\prime}} \mathbf{p}_{\mathbf{L}}$ belong to the same conjugacy class: $\sqrt{2 \alpha^{\prime}}\left(\mathbf{p}_{\mathbf{R}}-\mathbf{p}_{\mathbf{L}}\right) \in \Lambda_{\text {root }}$. Thus the above choice for $g_{a b}$ and $b_{a b}$ does indeed characterise a toroidal compactification on an EN lattice [13]. The boundary condition for open strings in the compact dimensions is,

$$
\left.\left(g_{a b} \partial_{\sigma} X^{b}-b_{a b} \partial_{\tau} X^{b}\right) \delta X^{a}\right|_{\sigma=0} ^{\sigma=\pi}=0,
$$

which can be satisfied by either Dirichlet conditions

$$
\left.\delta X^{a}\right|_{\sigma=0} ^{\sigma=\pi}=0
$$

or generalised Neumann conditions

$$
\left.\left[g_{a b} \partial_{\sigma} X^{b}-b_{a b} \partial_{\tau} X^{b}\right]\right|_{\sigma=0} ^{\sigma=\pi}=0
$$

Both conditions yield the same open string spectrum, a consequence of the E-duality generalisation of T-duality at the enhanced symmetry points of 
toroidal compactification. In terms of the Dirichlet winding $\mathbf{L}$ in Eq.(A.4) and of the Neumann momentum p, we have

$$
\begin{aligned}
\alpha^{\prime} m^{2} & =\frac{1}{\alpha^{\prime}} \mathbf{L} \cdot \mathbf{L}+N-1, \\
\text { or } \quad \alpha^{\prime} m^{2} & =\alpha^{\prime} \mathbf{p} \cdot \mathbf{p}+N-1, \\
\text { with } \quad \mathbf{L} & =\alpha^{\prime} \mathbf{p} .
\end{aligned}
$$

The last equation Eq.A.17) expresses the E-duality for open strings and shows that $\sqrt{2 \alpha^{\prime}} \mathbf{p}$ belongs to the root lattice.

\section{B Group-invariant twist operator}

For Neumann boundary conditions Eq. (A.14) we have

$$
\begin{aligned}
X^{a}(\sigma, \tau)=x^{a} & +g^{a c} b_{c b} B^{b} \sigma+B^{a} \tau+\sum_{n \neq 0} D_{n}^{a} \cos n \sigma \exp (-i n \tau) \\
& -\sum_{n \neq 0} i g^{a c} b_{c b} D_{n}^{b} \sin n \sigma \exp (-i n \tau) .
\end{aligned}
$$

The conjugate momentum Eq.(A.2) then takes the form

$$
\begin{aligned}
\Pi_{a}(\sigma, \tau) & =\frac{1}{2 \pi \alpha^{\prime}}\left[\left(g_{a b}-b_{a c} g^{c d} b_{d b}\right) B^{b}\right. \\
& \left.-i \sum_{n \neq 0} n\left(g_{a b}-b_{a c} g^{c d} b_{d b}\right) D_{n}^{b} \cos n \sigma \exp (-i n \tau)\right]
\end{aligned}
$$

To compute the commutation relations between the operators $x^{a}, B^{a}$ and

$D_{n}^{a}$ it is convenient to introduce the tensor $e_{a b} \equiv g_{a b}+b_{a b}$ and to define the E-dual metric $G^{a b}$ and antisymmetric tensor $B^{a b}$ by $\left(e^{-1}\right)^{a b} \equiv G^{a b}+B^{a b}$

$$
\left(g_{a c}+b_{a c}\right)\left(G^{c b}+B^{c b}\right)=\delta_{a}^{b} .
$$

This is equivalent to

$$
\begin{gathered}
g_{a b}-b_{a c} g^{c d} b_{d b}=\left(G^{-1}\right)_{a b}, \\
g^{a c} b_{c b}=-B^{a c}\left(G^{-1}\right)_{c b} .
\end{gathered}
$$


From Eqs.(B.18), (B.19) and (B.21) we get

$$
B^{a}=\left(2 \alpha^{\prime}\right) G^{a b} p_{b} \quad, \quad D_{n}^{a}=i \sqrt{2 \alpha^{\prime}} \frac{\alpha_{n}^{a}}{n},
$$

where the eigenvalues of the momentum operators $p_{b}$ are integers $m_{b}$. We rewrite $X^{a}$ in terms of the E-dual variables as

$$
\begin{aligned}
X^{a}=x^{a} & -2 \alpha^{\prime} B^{a b} p_{b} \sigma+2 \alpha^{\prime} G^{a b} p_{b} \tau+i \sqrt{2 \alpha^{\prime}} \sum_{n \neq 0} \frac{1}{n} \alpha_{n}^{a} \cos n \sigma \exp (-i n \tau) \\
& -\sqrt{2 \alpha^{\prime}} \sum_{n \neq 0} \frac{1}{n} B^{a c}\left(G^{-1}\right)_{c b} \alpha_{n}^{b} \sin n \sigma \exp (-i n \tau),
\end{aligned}
$$

where

$$
\left[\alpha_{m}^{a}, \alpha_{n}^{b}\right]=G^{a b} m \delta_{m+n, 0}
$$

The conventional twist operator is

$$
\Omega=e^{i \pi N} \quad, \quad N=\sum_{n>0}\left(G^{-1}\right)_{a b} \alpha_{-n}^{a} \alpha_{n}^{b}
$$

and gives

$$
\Omega X^{a}(\sigma ; B) \Omega^{-1}=X^{a}(\pi-\sigma ;-B)+\pi\left(2 \alpha^{\prime}\right) B^{a b} p_{b} .
$$

The matix elements $\left(2 \alpha^{\prime}\right) B^{a b}$ are, for EN lattices, \pm 1 or 0 and the eigenvalues of the last term in Eq. B.26) shifts $X^{a}(\pi-\sigma ;-B)$ to non equivalent lattice points. This effect is corrected by introducing a twist operator which is group invariant:

$$
\Omega^{\prime}=\Omega e^{i \pi \alpha^{\prime} \mathbf{p}^{2}}=e^{i \pi\left(N+\alpha^{\prime} \mathbf{p}^{2}\right)},
$$

where $\mathbf{p}$ is the Neumann momentum Eq. A.17) and $\mathbf{p}^{2}=p_{a} G^{a b} p_{b}$.

Eq. (B.26) becomes

$$
\Omega^{\prime} X^{a}(\sigma ; B) \Omega^{\prime-1}=X^{a}(\pi-\sigma ;-B)+\pi\left(2 \alpha^{\prime}\right)\left(e^{-1}\right)^{a b} p_{b} .
$$

The matrix elements $\left(e^{-1}\right)^{a b}$ are even integers and hence induce a shift to equivalent lattice points. Thus the group invariant operator $\left(1+\Omega^{\prime}\right) / 2$ is a valid projection operator onto unoriented open strings. Note that for $S U(2)$ $B^{a b}$ is zero and Eq.(B.26) makes sense. Nevertheless, the group invariant operator still induces a shift to equivalent lattice points. 


\section{Torus volumes}

Using Eqs.(A.5) and Eq.(A.6), we can compute $\sqrt{g}$ for EN lattices. We obtain

$$
\sqrt{g}=\left|\mathbf{e}_{\mathbf{a} 1} \wedge \mathbf{e}_{\mathbf{a} 2} \wedge \ldots \wedge \mathbf{e}_{\mathbf{a d}}\right|=\left(\frac{\alpha^{\prime}}{2}\right)^{d / 2} V_{\text {root }},
$$

where $V_{\text {root }}$ is the volume of the elementary cell of the $\mathcal{G}$ root lattice. It is equal to $\sqrt{\mathcal{N}}$ (see footnote 8 ). We thus get

$$
(2 \pi)^{d} \sqrt{g}=\left(2 \pi \alpha^{\prime 1 / 2}\right)^{d} \frac{\sqrt{\mathcal{N}}}{2^{d / 2}} .
$$

To evaluate $\sqrt{e}$, we notice using Eqs.(A.5), (A.7) that $e_{a b}$ is a triangular matrix. The determinant is given by the product of the diagonal elements $e_{a a}=\left(\alpha^{\prime} / 2\right) \mathbf{r}_{\mathbf{a}} \cdot \mathbf{r}_{\mathbf{a}}=\alpha^{\prime}$. We thus find

$$
(2 \pi)^{d} \sqrt{e}=\left(2 \pi \alpha^{\prime 1 / 2}\right)^{d}=V_{s d},
$$

which corresponds to the self-dual volume.

Therefore

$$
\sqrt{\frac{e}{g}}=\frac{2^{d / 2}}{\sqrt{\mathcal{N}}}
$$




\section{References}

[1] A. Casher, F. Englert, H. Nicolai and A. Taormina, Consistent Superstrings as Solutions of the $D=26$ Bosonic String Theory, Phys. Lett. B162 (1985) 121.

[2] P.G.O. Freund, Superstrings From Twenty-Six Dimensions?, Phys. Lett. B151 (1985) 387.

[3] P. Hasenfratz and G. 't Hooft, A Fermion-Boson Puzzle in a Gauge Theory, Phys. Rev. Lett. 36 (1976) 1119.

A.S. Goldhaber, Spin and Statistic Connection for Charge-Monopole Composites, Phys. Rev. Lett. 36 (1976) 1122.

[4] F. Englert, H. Nicolai and A.N. Schellekens, Superstrings from 26 Dimensions, Nucl. Phys. 274 (1986) 315.

[5] N. Seiberg and E. Witten, Spin Structures in String Theory, Nucl. Phys. 276 (1986) 272.

[6] L. Dixon and J. Harvey, String Theories in Ten Dimensions without Space-time Supersymmetry, Nucl. Phys. 274 (1986) 93.

L. Alvarez-Gaumé, P. Ginsparg, G. Moore and C. Vafa, An O(16) × O(16) Heterotic String, Phys. Lett. B171 (1986) 155.

H. Kawai, D. Lewellen and S. Tye, Classification of Closed Fermionic String Models, Phys. Rev. D34 (1986) 3794.

[7] W. Lerche, D. Lüst and A. Schellekens, Ten-Dimensional Heterotic Strings from Niemeier Lattices, Phys. Lett. B181 (1986) 71; Erratum, Phys. Lett. B184 (1987) 419.

[8] A. Schellekens and S. Yankielowicz, Curiosities at $C=24$, Phys. Lett. B226 (1989) 285.

A.N. Schellekens, Classification of Ten-Dimensional Heterotic Strings, Phys. Lett. B277 (1992) 277, hep-th/9112006.

[9] H. Nicolai and A.N. Schellekens, Topics in String Theory, in Proceedings of the 5th Adriatic Meeting on Superstrings, Anomalies and Unification, Dubrovnik (1986). 
[10] A. Sagnotti, in Non-Perturbative Quantum Field Theory, eds. G. Mack et al (Pergamon Press, 1988), p.521.

G. Pradisi and A. Sagnotti, Open String Orbifolds, Phys. Lett. B216 (1989) 59 .

M. Bianchi and A. Sagnotti, On the Systematics of Open String Theories, Phys. Lett. B247 (1990) 517.

M. Bianchi and A. Sagnotti, Twist Symmetry and Open-String Wilson Lines, Nucl. Phys. B361 (1991) 519.

A. Sagnotti, Some Properties of Open String Theories, hep-th/9509080.

A. Sagnotti, Surprises in Open-String Perturbation Theory, Nucl. Phys. Proc. Suppl. 56B (1997) 332, hep-th/9702093.

[11] M. Bianchi, G. Pradisi and A. Sagnotti, Toroidal Compactification and Symmetry Breaking in Open-String Theories, Nucl. Phys. B376 (1992) 365.

[12] K. Narain, New Heteroric String Theories in Uncompactified Dimensions < 10, Phys. Lett. B169 (1986) 41

K.S. Narain, M.H. Sarmadi and E. Witten, A note on Toroidal Compactification of Heterotic String Theory, Nucl. Phys. B289 (1987) 414.

[13] F. Englert and A. Neveu, Non Abelian Compactification of the Interacting Bosonic String, Phys. Lett. B163 (1985) 349.

[14] M. Halpern, Quantum Solitons which are su(n) Fermions, Phys. Rev. D12 (1975) 1684.

I. B. Frenkel and V. G. Kac, Basic Representations of Affine Lie Algebras and Dual Resonance Models, Inv. Math. 62 (1980) 23.

G. Segal, Unitary Representations of Some Infinite Dimensional Groups, Comm. Math. Phys. 80 (1981) 301.

[15] F.A. Bais, F. Englert, A. Taormina and P. Zizzi, Torus Compactification for Non Simply Laced Group , Nucl. Phys. 279 (1987) 529.

[16] W. Lerche, A. Schellekens and N.P. Warner, Lattices and Strings, Physics Reports 177 (1989) 1-140.

[17] A. Schellekens, Multiloop Modular Invariance of the Covariant Lattice Construction od Fermionic Strings, Phys. Lett. B199 (1987) 427. 
[18] M. Douglas and B. Grinstein, Dilaton Tadpole for the Open Bosonic String, Phys. Lett. B183 (1987) 552; Erratum B187 (1987) 442.

$\mathrm{S}$. Weinberg, Cancellation of the one loop divergences in SO(8192) string theory, Phys. Lett. B187 (1987) 278.

N. Marcus and A. Sagnotti, Group Theory from 'Quarks' at the ends of strings, Phys. Lett. B188 (1987) 58.

M. Bianchi and A. Sagnotti, The Partition Function of the $\mathrm{SO}(8192)$ bosonic string, Phys. Lett. B211 (1988) 407.

[19] C. G. Callan, C. Lovelace, C. R. Nappi and S. A. Yost, Adding Holes and Crosscaps to the Superstrings, Nucl. Phys. B293 (1987) 83.

C. G. Callan, C. Lovelace, C. R. Nappi and S. A. Yost, Loop Corrections to Superstring Equations of Motion, Nucl. Phys. B308 (1988) 221.

[20] J. Polchinski and Y. Cai, Consistency of Open Superstring Theories, Nucl. Phys. B296 (1988) 91.

[21] W. Fishler and L. Susskind, Dilaton Tadpoles, String Condensates and Scale Invariance, Phys. Lett. B171 (1986) 383.

W. Fishler and L. Susskind, Dilaton Tadpoles, String Condensates and Scale Invariance 2, Phys. Lett. B173 (1986) 262.

[22] J. Polchinski, String Theory, Vol. 1, Cambridge Univ. Press, 1998.

[23] G. Pradisi, A. Sagnotti and Ya. S. Stanev, Planar Duality in SU(2) WZW models, Phys. Lett. B273 (1995) 279,hep-th/9503217.

G. Pradisi, A. Sagnotti and Ya. S. Stanev, The Open Descendants of Non-Diagonal SU(2) WZW Models, Phys. Lett. B356 (1995) 230, hep-th/9506014.

[24] O. Bergman and M. R. Gaberdiel, Dualities of Type O Strings, J. High Energy Phys. 9907 (1999) 22, hep-th/9906055.

[25] J. Polchinski, TASI Lectures on D-branes, hep-th/9611050.

[26] E. S. Fradkin and A. Tseytlin, Non-Linear Electrodynamics from Quantized Strings, Phys. Lett. B163 (1985) 123.

[27] R. G. Leigh, Dirac-Born-Infeld Action for Dirichlet $\sigma$-Models, Mod. Phys. Lett. A4 (1989) 2767. 
[28] J. A. Harvey, S. Kachru, G. Moore and E. Silverstein, Tension is Dimension, JHEP 0003 (2000) 01, hep-th/9909072.

[29] I. R. Klebanov and A. A. Tseytlin, D-branes and Dual Gauge Theories in type O String Theory, Nucl. Phys. B546 (1999) 155, hep-th/9811035.

[30] G.T. Horowitz and L. Susskind, Bosonic M Theory, hep-th/0012037.

[31] S. Elitzur, E. Gross, E. Rabinovici and N. Seiberg, Aspects of Bosonization in String Theory, Nucl. Phys. B283 (1987) 413. 